\title{
Quality of Service Shared Cache Management in Chip Multiprocessor Architecture
}

\author{
FEI GUO \\ VMware, Inc. \\ YAN SOLIHIN \\ North Carolina State University \\ and
LI ZHAO and RAVISHANKAR IYER
Intel Corporation

The trends in enterprise IT toward service-oriented computing, server consolidation, and virtual computing point to a future in which workloads are becoming increasingly diverse in terms of performance, reliability, and availability requirements. It can be expected that more and more applications with diverse requirements will run on a Chip Multi-Processor (CMP) and share platform resources such as the lowest level cache and off-chip bandwidth. In this environment, it is desirable to have microarchitecture and software support that can provide a guarantee of a certain level of performance, which we refer to as performance Quality of Service.

In this article, we investigated a framework would be needed to manage the shared cache resource for fully providing QoS in a CMP. We found in order to fully provide QoS, we need to specify an appropriate QoS target for each job and apply an admission control policy to accept jobs only when their QoS targets can be satisfied. We also found that providing strict QoS often leads to a significant reduction in throughput due to resource fragmentation. We proposed throughput optimization techniques that include: (1) exploiting various QoS execution modes, and (2) a microarchitecture technique, which we refer to as resource stealing, that detects and reallocates excess cache capacity from a job while preserving its QoS target. We designed and evaluated three

This work is supported in part by the National Science Foundation through grant CNS-0406306 and CCF-0347425, and by gifts from Intel.

Much of the work was performed when F. Guo was a Ph.D. student at NCSU.

An early version of this article titled "A Framework for Providing Quality of Service in Chip MultiProcessors" appeared in Proceedings of the 40th Annual IEEE / ACM International Symposium on Microarchitecture [Guo et al. 2007].

Authors' addresses: F. Guo, VMware Inc., 3401 Hillview, Sunnyvale, CA 94304; email: fguo@vmware.com; Y. Solihin, North Carolina State University, Raleigh, NC 27695-7256; email: solihin@ncsu.edu; L. Zhao and R. Iyer, Intel Corporation, Hillsboro, OR 97124; email: \{li.zhao, ravishankariyer\}@intel.com.

Permission to make digital or hard copies of part or all of this work for personal or classroom use is granted without fee provided that copies are not made or distributed for profit or commercial advantage and that copies show this notice on the first page or initial screen of a display along with the full citation. Copyrights for components of this work owned by others than ACM must be honored. Abstracting with credit is permitted. To copy otherwise, to republish, to post on servers, to redistribute to lists, or to use any component of this work in other works requires prior specific permission and/or a fee. Permissions may be requested from Publications Dept., ACM, Inc., 2 Penn Plaza, Suite 701, New York, NY 10121-0701 USA, fax +1 (212) 869-0481, or permissions@acm.org. (C) 2010 ACM 1544-3566/2010/12-ART14 $\$ 10.00$

DOI 10.1145/1880037.1880039 http://doi.acm.org/10.1145/1880037.1880039

ACM Transactions on Architecture and Code Optimization, Vol. 7, No. 3, Article 14, Pub. date: December 2010. 
algorithms for performing resource stealing, which differ in how aggressive they are in stealing excess cache capacity, and in the degree of confidence in meeting QoS targets. In addition, we proposed a mechanism to dynamically enable or disable resource stealing depending on whether other jobs can benefit from additional cache capacity. We evaluated our QoS framework with a full system simulation of a 4-core CMP and a recent version of the Linux Operating System. We found that compared to an unoptimized scheme, the throughput can be improved by up to $47 \%$, making the throughput significantly closer to a non-QoS CMP.

Categories and Subject Descriptors: C.1.4 [Computer Systems Organization]: Parallel Architecture; C.4 [Computer Systems Organization]: Performance of System

General Terms: Design, Performance

Additional Key Words and Phrases: Cache, chip multi-processors, CMP, multicore architecture, quality of service, QoS, performance, resource stealing

ACM Reference Format:

Guo, F., Solihin, Y., Zhao, L., and Iyer, R. 2010. Quality of service shared cache management in chip multiprocessor architecture. ACM Trans. Architec. Code Optim. 7, 3, Article 14 (December 2010), 33 pages. DOI = 10.1145/1880037.1880039 http://doi.acm.org/10.1145/1880037.1880039

\section{INTRODUCTION}

Recently, Chip Multi-Processor (CMP) or multicore design has become the mainstream architecture of choice for major microprocessor makers. Compared to single core design, CMPs provide throughput improvement for multithreaded and multiprogrammed workloads. However, since many on-chip platform resources, such as the lowest level on-chip cache and off-chip bandwidth, are shared by all the processor cores, the performance and throughput of programs running on a CMP depend heavily on how these resources are allocated to them. The absence of resource allocation policies and the inability to partition these resources can lead to severe resource contention and result in a large performance variation for many applications [Chandra et al. 2005; Hsu et al. 2006; Iyer 2004; Iyer et al. 2007; Kim et al. 2004; Suh et al. 2001]. As the number of cores in a CMP increases, the degree of sharing of platform resources can be expected to increase and will only exacerbate the performance variability problem.

A large performance variation suffered by applications in current CMPs is ill-suited for future uses of CMPs considering recent trends in enterprise IT toward service-oriented computing, server consolidation, and virtualization. These trends point to a future in which CMPs will run a diverse set of applications that have diverse computing requirements [Ranganathan and Jouppi 2005]. In these environments, many applications will require a guarantee of a certain level of performance, and we refer to such a guarantee as performance Quality of Service (QoS). For example, in a service-oriented or utility computing environment, the utility computing provider may set up different service-level agreements (SLAs) to different clients that encapsulate guarantees in performance, reliability, manageability, and other metrics [Papazoglou and Georgakopoulos 2003; Ranganathan and Jouppi 2005]. Such an environment would require the server to be able to allocate platform resources proportional to the level of the performance guarantee for each workload. For example, a job 


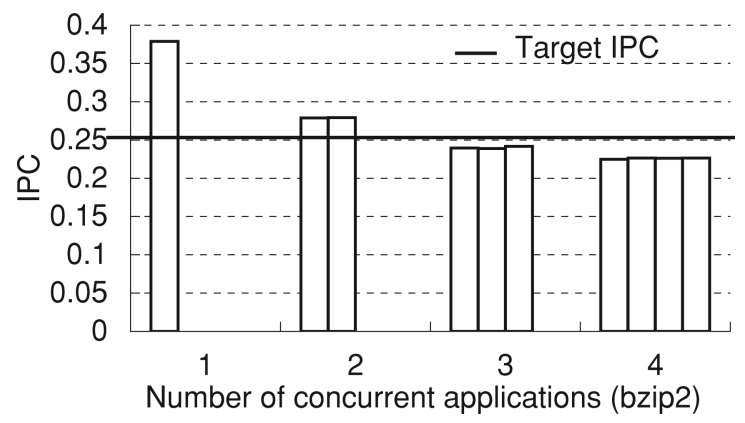

Fig. 1. The IPC of different numbers of instances of bzip2 running on a 4-core CMP with a 32-KB L1 cache per core, and a 2MB L2 cache shared by all cores. The full evaluation setup can be found in Section 7.

from a client with a "gold" SLA may be allocated more resources (e.g., larger cache sizes, higher processor count, and off-chip bandwidth) compared to jobs with standard SLAs. In virtualization, a virtual machine manager (VMM) hosts multiple virtual machines (VMs), where each VM runs a guest operating system for various purposes ranging from regular to critical computations. It is beneficial if the VMM can allocate more platform resources to critical VMs and fewer resources to regular VMs. Finally, many transaction processing applications in a service-oriented computing domain would require a minimum level of real-time performance to be guaranteed. Overall, we believe that it is critical to support QoS in CMPs.

In recent studies, researchers have introduced frameworks in which applications specify their QoS targets, expressed in instructions per cycle (IPC) or resource performance (e.g., cache miss rates), while a resource manager dynamically partitions shared resources in order to meet each application's QoS target [Hsu et al. 2006; Iyer 2004; Iyer et al. 2007; Nesbit et al. 2007; Rafique et al. 2006]. Unfortunately, these frameworks are insufficient if one wants to fully provide QoS in CMPs. Figure 1 shows an example in which multiple jobs (each job runs the SPEC2006 benchmark bzip2) run on a 4-core CMP with private L1 caches but a shared L2 cache. Let us assume that each job's QoS target is to reach an IPC of at least 0.25 , which is $\frac{2}{3}$ of its IPC when it runs alone. If the resource manager tries to satisfy the QoS targets of all jobs, it will equally divide the L2 cache among all instances of bzip2. However, from this figure, we can see that while the jobs' QoS targets are met when only two jobs run simultaneously, they are not met when three or four jobs run in the CMP. There are two major reasons why such frameworks fail in meeting the QoS targets of the jobs. First, the CMP does not have the ability to check whether its available resources are sufficient to satisfy a job's IPC target or the amount of resources needed by the job to meet its IPC target. Secondly, the lack of an admission control policy means that jobs are always accepted and run even when their QoS targets cannot be met.

Contributions. In this article, we investigate a framework for providing QoS in a CMP system. While the framework is applicable for managing various 
platform resources, in this study we limit our investigation to managing the shared last level cache.

First, we found that popular metrics (IPC and miss rate) are not suitable for specifying a QoS target, while capacity specification (e.g., cache size) naturally allows comparing the available computation capacity versus the demanded capacity. This enables us to build an admission control policy to ensure that jobs are only accepted when their QoS targets can be satisfied.

Second, we introduce three $Q o S$ execution modes, as a way for jobs to specify how flexible they are with regard to their QoS targets. These execution modes are needed to match users' diverse requirements for their workloads as well as to provide a means for the system to boost throughput.

We applied our framework for a CMP and found that providing a strict QoS guarantee usually comes at a cost of significant reduction in system throughput due to the cache resource fragmentation when jobs overspecify their QoS targets. We propose and investigate two techniques to recover the lost throughput. The first technique speculatively downgrades a job's QoS execution mode in order to boost the overall throughput. The second is a microarchitecture technique that we refer to as resource stealing, which removes excess cache capacity from a job without violating the job's QoS target. To achieve resource stealing, we propose and evaluate three algorithms that differ in how aggressive they are in stealing excess cache capacity and in the degree of confidence in meeting QoS targets. In addition, we proposed a mechanism to dynamically enable or disable resource stealing depending on whether other jobs can benefit from additional cache capacity.

We evaluate the proposed schemes and mechanism on a 4-core CMP machine model based on Simics, with a recent version of the Linux Operating System, and workloads constructed by using SPEC2006 benchmarks. We found that through a combination of appropriate QoS target specification, admission control, and execution modes, a CMP can ensure that all accepted jobs have their QoS targets satisfied. In addition, we found that the proposed QoS execution modes and resource stealing technique are effective in improving throughput without violating jobs' QoS targets. They achieve throughput improvement between $13 \%$ and $47 \%$, making the throughput significantly closer to a non-QoS CMP.

The rest of the article is organized as follows. Section 2 discusses related work. Section 3 presents QoS target specification and execution modes. Section 4 presents our resource stealing techniques for improving throughput. Section 5 presents the dynamic resource stealing mechanism. Section 6 discusses the implementation of an admission control policy. Section 7 presents the evaluation setup, while Section 8 presents and discusses the evaluation results. Finally, Section 9 summarizes the findings.

\section{RELATED WORK}

In a CMP system, some platform resources, such as the off-chip bandwidth and the lowest level on-chip cache, are typically shared among cores. With the increasing number of cores on a chip (possibly to more than one hundred cores 
by 2015 [Borkar et al. 2005]), the contention for these critical shared resources suffered by applications running simultaneously in different cores will increase significantly and needs to be carefully managed.

Some studies that address the management of shared resources have focused on improving the overall throughput or fairness of the CMP. Suh et al. [2001] proposed a cache partitioning policy that minimizes the total number of cache misses, Kim et al. [2004] proposed a cache partitioning policy that optimizes for uniform slowdown (fairness) to applications that share the cache, while Qureshi and Patt [2006] proposed utility-based cache partitioning. Hsu et al. [2006] studied the impact of various optimization goals in guiding how cache partitions are allocated in a CMP architecture. The optimization goals include maximizing the overall performance metric (e.g., IPC or miss rate) and the overall fairness. Cho and Jin [2006] proposed an OS-level page allocation algorithm in a shared L2 non-uniform cache architecture for future many-core processors to reduce the cache access latency, on-chip network traffic and power consumption. Chang and Sohi [2007] proposed a cooperative cache partitioning algorithm that optimizes for several metrics, such as the sum of per-thread slowdowns as well as the harmonic mean of per-thread speedups over an equalpartition cache baseline. While these studies seek to mitigate the impact of contention and optimize for an overall goal, they do not provide QoS to individual applications.

Some recent studies have recognized the need for CMPs to have QoSenabling features to provide differentiated services to various applications. For example, Iyer [2004] described the need for a priority-based QoS framework in CMP architectures in which a job can specify whether it should be run with a high or low priority, and resource allocation is guided by job priorities. Rafique et al. [2006] proposed an architecture support and Operating System (OS) interface that allows OS-level cache partitioning, in which an application is prevented from occupying more than a certain fraction (quota) of the cache. Nesbit et al. [2007] proposed a Virtual Private Cache (VPC) that combines the resource allocation policies for caches and the memory controller using a fair queuing algorithm. VPC provides an abstraction of private caches through partitioning the shared cache into per-core partitions, while resource allocation policies ensure that the IPC achieved is equal to that of real private caches. Iyer et al. [2007] proposed several priority-based resource management policies as well as a QoS-aware cache and memory architecture. Individual applications can specify their own QoS target (e.g. IPC, miss rate, cache space) and the hardware dynamically adjusts cache partition sizes to meet their QoS targets. Lin et al. [2008] proposed a software-based experimental methodology to implement and evaluate several cache partitioning policies that optimize for fairness and QoS. The OS uses page allocation in order to perform dynamic cache partitioning to achieve applications' IPCs. We note that while all above studies mention QoS, they do not associate QoS with the notion of performance guarantee. While both priorities and cache quota correlate with performance and help the system to favor one application over another in allocating resources, they do not automatically provide QoS guarantees to the applications.

ACM Transactions on Architecture and Code Optimization, Vol. 7, No. 3, Article 14, Pub. date: December 2010. 


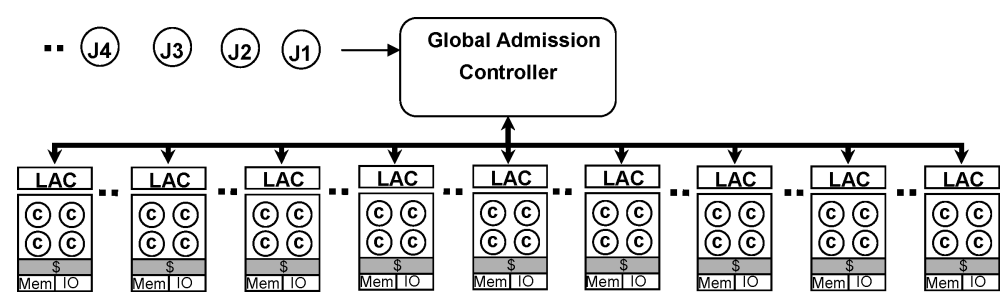

Fig. 2. The assumed working environment.

In some QoS models, individual applications can specify an acceptable performance level expressed in IPC. The system then translates the IPC into the minimum resources required to achieve it, through profiling a thread at runtime and recording how the thread's IPC changes as the amount of resources are varied [Lee and Asanovic 2006; Yeh and Reinman 2005]. The fact that the studies above require a greedy search in the resource allocation space illustrates the difficulty of using IPC as a QoS target. We believe that to really provide QoS, the CMP must have an ability to easily compare available and demanded computation capacity, and such ability is a critical component that enables the construction of an admission control policy.

Finally, some of the concepts in our framework, such as the resource reservation and admission control, are borrowed from the real-time system domain [Brandenburg and Anderson 2007; Buttazzo et al. 2002; Rajkumar et al. 1998]. However, while in traditional real-time systems the operating system and processor architecture are structured to suit the needs of real-time constraints, we seek to provide QoS in CMP-based general purpose servers with a largely unmodified OS, processor architecture, and memory hierarchy.

\section{QOS TARGET SPECIFICATION AND EXECUTION MODES}

This section presents the assumptions of our working environment (Section 3.1), QoS goals and metrics for specifying a QoS target (Section 3.2), and the proposed QoS execution modes (Section 3.3).

\subsection{Definitions and Assumptions}

We refer to a job as the unit of aperiodic computation task that has its own QoS target. A job may consist of a thread, an application, or a group of applications. In this article, we limit our study by associating an instance of a single-threaded application as a job. We define computation capacity as the resources that can be used for providing performance. Basically, a job's QoS target is computation capacity demand, while available resources in the server are computation capacity supply.

We assume a server platform consisting of CMP nodes as shown in Figure 2. The server has a global admission controller (GAC), which decides whether to accept or reject a newly arriving job submitted by user. To achieve this, the global admission controller probes each CMP node's local / per-CMP admission controller (LAC) to find which CMP node can accept the job and satisfy its QoS target. When the GAC cannot find any CMP node that can accept the job, it 
rejects this job or negotiates with the user for another acceptable QoS target. A comprehensive discussion of the GAC is beyond the scope of this article. In this article, only the LAC is considered as a component of our QoS framework because it has a direct interaction with microarchitecture resources.

\subsection{Specifying QoS Target}

Earlier we have argued that for a CMP to fully provide QoS to an incoming job, it must have available computation capacity in excess of the capacity demanded by the job. We refer to the units of target as the units in which a QoS target is specified, and units of capacity as the units in which computation capacity in the CMP is expressed. Before continuing our discussion, it is helpful to define one term.

Definition 1. A QoS target is convertible if its units of target can be converted into units of computation capacity.

In order for a CMP to really provide QoS, two conditions must be met. The first condition is that a QoS target must be convertible, which allows the system to easily compare the available computation capacity with demanded capacity. The second condition is that a job should be accepted only if its QoS target can be satisfied. Convertibility and satisfiability checking are the basis for constructing an admission control policy to ensure that the QoS targets of all accepted jobs can be met.

One way to provide QoS in a CMP system is to model it after a traditional real-time system in which the QoS target of a job is specified by its deadline. In a real-time system, deadline convertibility can be achieved through Worst-Case Execution Time (WCET) analysis which determines the maximum execution time of a job by taking into account the maximum path length of the code and maximum latencies that can occur in the architecture. Unfortunately, in traditional real-time systems the operating system and processor architecture are often structured to suit the needs of real-time constraints, such as by restricting out-of-order execution, dynamic branch prediction, and a complex memory hierarchy. Our goal is to provide QoS in general purpose servers with a largely unmodified OS, processor architecture, and memory hierarchy. Furthermore, in a server environment, jobs often have unpredictable arrivals, dynamic and input-dependent behavior, and may not have meaningful deadlines. Hence, unlike in traditional real-time systems, we cannot use deadlines as the primary QoS target.

Iyer et al. [2007] proposed three types of QoS targets. The first is $R e$ source Usage Metrics (RUM), which specify the amount of resources needed by the application, such as the processor count, cache size, and bandwidth rate. The second is Resource Performance Metrics (RPM), which specify the performance of specific resources being used, for example cache miss rates. The final is Overall Performance Metrics (OPM), which specify the overall throughput of the program, expressed in IPC. Most prior studies in architecture support for QoS assume that the QoS target is expressed in IPC. However, we believe that IPC is not suitable to specify a QoS target because IPC is not easily 
convertible. A CMP system cannot easily determine how much IPC it can provide for a particular job (unless it uses an elaborate performance model). Furthermore, it also cannot easily determine the amount of platform resources that are needed to achieve a target IPC. Similarly, a CMP cannot easily determine what miss rate it can provide to a particular job or the amount of resources needed in order to provide a given miss rate. In fact, in addition to being non-convertible, it may be hard to check whether particular RPM and OPM values are not ill-defined, that is, they cannot be satisfied no matter how many resources are allocated. As a result, we believe that OPM and RPM are not suitable for a CMP system to fully provide QoS.

In contrast to RPM and OPM, RUM are easily convertible if a CMP is equipped with relatively simple hardware that tracks the current allocation of platform resources for different cores. For example, with RUM, an incoming job's request in terms of the amount of cache capacity it needs (demand) can be compared trivially against the amount of cache capacity that has not been allocated yet (supply). This leads to the ease of constructing an admission control policy. An additional benefit of using RUM is that such metrics are already used in batch job systems. For example, in the Lsbatch batch job system [Wang et al. 1993], a job can specify its requirements in terms of the number of processors, memory size, disk space size, and the maximum wall clock time it would run. Hence, RUM has an advantage of being time-tested and has a high familiarity with users.

In the context of CMPs, RUM must extend beyond resources that are specified in traditional batch job systems, for example by including the shared cache capacity and off-chip bandwidth rate. In this work, we focus on the shared L2 cache capacity and processor core resources in the QoS target specification. We acknowledge that a complete QoS specification would include off-chip bandwidth rate, main memory size, network bandwidth, disk size, and other resources. However, we limit our study to the shared last level cache, and leave the simultaneous management of multiple resources for future work.

Optionally, a QoS target may include a timeslot resource, which can be specified through a maximum wall-clock time which indicates the size of the timeslot, and a deadline which indicates the latest expected completion of the timeslot. Maximum wall-clock time is a concept borrowed from batch job systems [Wang et al. 1993]. It specifies the maximum amount of time that a job should be allowed to run assuming it gets all its requested resources. The maximum wall-clock time is different from WCET in a real-time system in that it does not need to be a safe execution time upper bound. Embedded in it is the users' expectation that a job may be terminated if it runs longer than its maximum wall-clock time.

In order to make it easier for users to determine an appropriate QoS target, the system may provide a profiling tool that can suggest good resource amounts to specify initially [Lee and Asanovic 2006]. Alternatively, the system may provide several preset RUM targets that users can choose from. Similar preset targets have been employed in many batch job systems. For example, a job can choose one of large, medium, or small configurations. Each configuration comes with preset memory size, maximum processor count, and maximum wall-clock 
time. A reasonable default configuration to use is one in which all resources are partitioned equally among all cores. However, while preset QoS targets could greatly simplify QoS target selection for users, they may also exacerbate QoS overspecification, a situation in which a job needs less resources than what it specifies. This leads to resource fragmentation that will reduce overall throughput. We will address how to recover from resource fragmentation in the following sections.

\subsection{QoS Execution Modes}

Besides QoS target specification, another important component of our QoS framework is how strictly the QoS target must be followed. Similar to the postal delivery system that allows various strictness levels in delivery times, it may be desirable for a utility computing server to provide various strictness levels in meeting the QoS target. To achieve this variety, we propose the following execution modes.

(1) Strict. The Strict execution mode may be used by jobs that have rigid requirements for a minimum throughput (implied by the RUM) and deadline. To meet a Strict job's QoS target, the requested resources and timeslot must be strictly reserved.

(2) Elastic $(X) .{ }^{1}$ The Elastic(X) execution mode can be used by jobs that have a strict deadline requirement but can tolerate some deviation of throughput compared to that implied by the amount of resources requested in RUM. The deviation is such that the reduction of throughput (slowdown) is not more than X\% compared to the case in which the resources are reserved (i.e., in the Strict mode).

(3) Opportunistic. The Opportunistic mode may be used by jobs that do not have rigid throughput and deadline requirements. For example, users may use the Opportunistic mode for jobs whose deadlines are still far away.

It is likely that in many environments, most jobs are submitted in Elastic(X) and Opportunistic modes, with X likely being a small number such as 5-10\%. Strict jobs are probably only used for applications which must achieve a certain level of throughput to perform acceptably, or for applications that are timecritical and have deadlines.

It is important to note that Strict and Elastic $(\mathrm{X})$ modes do not require a job's deadline to be specified, as it is an optional QoS target. Some types of jobs may not have meaningful deadlines, such as long-running jobs, OS daemons, or some legacy applications. For these jobs, the specified resources will be reserved for their entire lifetime. In other words, job deadline impacts only the scheduling decision rather than the enforcement of QoS execution modes.

Note that since we propose to use RUM in our QoS target specification, the Elastic(X) mode is only meaningful when $\mathrm{X}$ is defined with a different metric (not a RUM). Otherwise, Elastic(X) mode will be equivalent to the Strict mode

\footnotetext{
${ }^{1}$ The concept of Elastic mode is similar to the one in Buttazzo et al. [2002]. However, while Buttazzo et al. [2002] it only applies to periodic jobs, we define the elasticity in terms of slowdown, hence it is applicable to aperiodic jobs as well.
}

ACM Transactions on Architecture and Code Optimization, Vol. 7, No. 3, Article 14, Pub. date: December 2010. 
with a reduced resource requirement. As a result, for the Elastic(X) execution mode, we specify $\mathrm{X}$ in terms of the percentage of slowdown in execution time or cycle-per-instruction (CPI). From users' point of view, the significance of the availability of the weaker modes (Elastic $(\mathrm{X})$ and Opportunistic) is that they may match their jobs' requirements better, especially if the weaker modes have lower fee structures. From the system point of view, we will show that weaker modes allow the CMP to boost throughput by accepting more jobs and mitigating QoS target overspecification.

In the context of utility computing, the QoS execution modes can be integrated into Service Level Agreements (SLAs) and can be associated with a certain fee structure. For example, users may pay more to run jobs in a Strict mode but enjoy a strong guarantee that the requested resources are allocated to it, or pay less to run jobs in the Elastic(X) mode or Opportunistic mode. From the point of view of service providers, they can improve revenue by maximizing the number of jobs that the system can accept and simultaneously run. In such an environment, resources are scarce and need to be allocated efficiently. Hence, we do not address policies to handle idle resources.

Downgrading QoS Execution Mode. Suppose a user submits a job in Strict mode, and the CMP rejects the job because it cannot meet the QoS target of the job. In this case, the user can probably make the job admissible if he/she is willing to reduce the required resources specified in the QoS target, increase the deadline, or downgrade the mode of their job to a weaker one. We refer to changing the mode from Strict to Elastic(X) or Opportunistic, or from Elastic(X) to Opportunistic by users as manual mode downgrade. Since users are fully aware of the consequence of using different execution modes, manual mode downgrade requires the willingness of users to reduce their expectations on the strictness of the jobs' QoS targets.

Mode downgrade can also be performed transparently by the system as long as the old and new modes are interchangeable. We define two modes as interchangeable if they can be used to guarantee completion of a job by the same deadline, and throughput variation can be tolerated by the job. Suppose we have a Strict job with deadline of $t d$ and maximum wall-clock time of $t w$, arriving at time $t a$. We note that there is a time slack of $(t d-t a)-t w$. This amount of slack means that the job can be downgraded as an Elastic $\left(\frac{(t d-t a)-t w}{t w}\right)$ job while still meeting its deadline. Additionally, it can also be downgraded to the Opportunistic mode for $(t d-t a)-t w$ amount of time, but if it has not completed by time $t d-t w$, it needs to be switched back to the Strict mode. We refer to these as automatic mode downgrade.

\subsection{The Impact of Execution Mode Downgrade}

In this section, we examine the impact of manual and automatic mode downgrade. Before we continue the discussion, it is helpful to distinguish two factors that contribute to suboptimal throughput when there are only Strict jobs. The first is external resource fragmentation, which refers to idle resources that are not allocated to any jobs. Typically, external resource fragmentation occurs when there are not sufficient remaining available resources to accept a new 


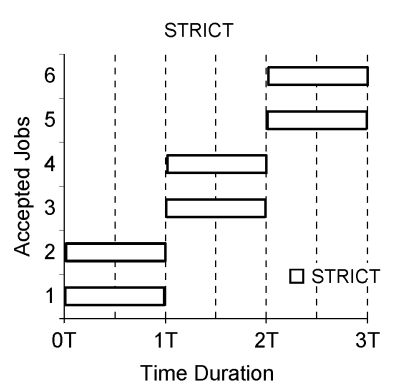

(a)

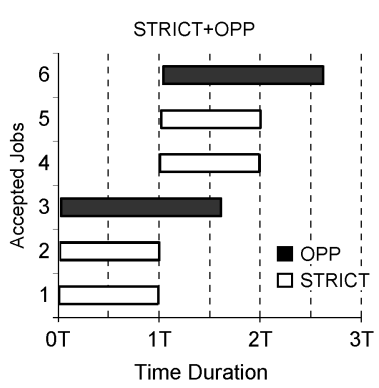

(b)

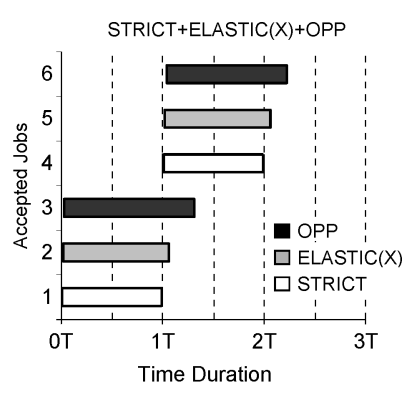

(c)

Fig. 3. Illustration of the impact of manual mode downgrade: all Strict jobs (a), third and sixth accepted jobs are manually downgraded to Opportunistic (b), and second and fifth accepted jobs are downgraded to Elastic(X) (c).

job. The second factor is internal resource fragmentation, which is caused by a job not using all resources that are allocated to it.

First, let us consider the impact of manual mode downgrade, as illustrated in Figure 3. In the figure, each bar represents the time each job takes to complete its computation, the $\mathrm{x}$-axis represents the time duration and the $\mathrm{y}$-axis represents different accepted jobs. In this example, we assume that jobs are sequentially submitted to the system, and the first six accepted jobs are shown in the figure. Assume that each job requires $40 \%$ of the shared cache size in order to complete in $\mathrm{T}$ time. The deadline of each job is assumed to be $1.5 \mathrm{~T}$ from the time when the job is accepted. If there are six Strict jobs (Figure 3(a)), at most two jobs can be executed simultaneously since there is not enough cache space to run more than two jobs simultaneously. The external resource fragmentation includes two idle cores in a 4-core CMP and 20\% of the L2 cache capacity. The CMP takes $3 \mathrm{~T}$ to finish all six jobs and all of them meet their deadlines. However, if users manually downgrade the third and sixth jobs to Opportunistic jobs (Figure 3(b)), the system can accept and run more jobs simultaneously and reduce external resource fragmentation. Although the third and the sixth jobs run slower, the overall throughput is improved as it only takes slightly more than $2.5 \mathrm{~T}$ to complete all six jobs. If users also manually downgrade the second and fifth jobs to Elastic(X) jobs, the system can employ the resource stealing technique (Section 4 ) to discover unused cache capacity allocated to Elastic $(\mathrm{X})$ jobs and reallocate them to Opportunistic jobs. This results in the third and sixth jobs completing faster, while the second and fifth jobs run slower but still meet their deadlines. The overall throughput is potentially improved further as we also reduce internal cache fragmentation.

With manual mode downgrade, when a Strict job is downgraded to Elastic(X), its unused resources are reallocated to Opportunistic jobs. However, since the job may be slowed down by up to X\%, in order to guarantee meeting its deadline, the job needs to reserve resources for a longer time duration of $t w \times(1+X)$ (versus $t w$ if it remains a Strict job). Since the same amount of resources are reserved for a longer time, the ability of the CMP to accept future jobs may be reduced, which in turn may reduce future throughput. Consequently, manual downgrade of a Strict job to Elastic(X) may reduce throughput if it is not 
accompanied by a throughput increase due to Opportunistic jobs benefiting from reallocation of excess resources. Overall, we can expect throughput improvement to be higher when there are both Elastic(X) and Opportunistic jobs to complement Strict jobs, compared to when there are only Opportunistic jobs to complement Strict jobs.

With automatic mode downgrade, an additional impact occurs when a Strict job is downgraded to Opportunistic mode. In contrast to the manual mode downgrade in which an Opportunistic job does not reserve any resources, with automatic mode downgrade the resources requested by the job still need to be reserved for the length of its maximum wall-clock time. The job can only be run in Opportunistic mode before it meets its reserved timeslot, by which time it has to switch back to Strict mode in order to ensure that its deadline is met. When a job completes before it meets its reserved timeslot, the reserved resources can be reclaimed to allow new jobs to be accepted and future throughput to be improved. As a result, the reserved timeslot needs to be placed as far away as possible in order to increase the probability that the job completes before the reserved timeslot is encountered. Finally, we do not consider automatically downgrading a Strict job to Elastic(X) job because the Elastic(X) mode reserves resources longer than the original Strict mode, which is likely to be detrimental to throughput.

Comparing manual and automatic mode downgrade, if the manually downgraded Opportunistic jobs do not suffer from too much slowdown due to lack of resources, it can be expected that manual downgrade would achieve a higher throughput than automatic downgrade since the latter still relies on resource reservation, which may reduce job admission rate. However, the automatic mode downgrade is still useful because it does not rely on users' willingness to downgrade their jobs to weaker modes.

\section{RESOURCE STEALING TECHNIQUES}

In this section, we will discuss resource stealing, a key technique that supports the proposed Elastic $(\mathrm{X})$ execution mode.

\subsection{Managing Cache Capacity Partitions}

In order to track and control the shared cache allocation across cores, we need to employ a cache partitioning scheme. Our cache partitioning scheme is based on a fine-grain modified LRU replacement policy in Iyer [2004] and Nesbit et al. [2007], but we adapt it to our QoS framework. In Iyer [2004] and Nesbit et al. [2007], each core is assigned a target allocation counter that records the number of cache ways that should be assigned to it. Each set in the cache also has per-set counter to track the number of blocks in the set that are currently allocated to the core. When a core suffers a cache miss on a set, the core's per-set counter is compared against its target allocation counter. If the per-set counter has a lower value, a block that belongs to one of the overallocated cores is selected as the victim. Otherwise, a block that belongs to the core itself is selected as the victim. After a sufficient amount of time, the number of blocks allocated to a core will be the same over all sets. 
In our QoS framework, each processor core is augmented with an extra register which records the execution mode of the currently running job. This register is treated as a part of the context of the application, that is, it is saved and restored at the context switch boundary. With this hardware support, the selection of the victim block for a cache miss also depends on the execution mode of the job that the victim belongs to. On each cache miss, if there is more than one overallocated core, the victim is first selected from an overallocated Strict or Elastic(X) job (if there is any). Otherwise, the LRU block among the blocks from Opportunistic jobs is selected as the victim. The reason why overallocated Strict/Elastic $(\mathrm{X})$ jobs are given a higher priority for victim selection is that we would like to accelerate the cores running these jobs in converging to their target allocations, and reallocate the excess cache capacity from Elastic $(\mathrm{X})$ jobs to Opportunistic jobs as fast as possible.

\subsection{Criteria for Resource Stealing}

In Section 3.3, the Elastic(X) execution mode was proposed in order to enable the system to remove excess cache capacity allocated to a job due to QoS ovespecification, and X specifies the maximum slowdown (slack) that is acceptable to the user. When applying resource stealing, it is difficult to accurately measure how much CPI increases when we employ resource stealing versus when we do not. Hence, we need a more measurable metric.

First, we note that components of CPI are additive, that is, the overall CPI is the sum of CPI assuming an infinite cache and additional CPI when cache misses are considered [Emma 1997; Luo et al. 1998]. Specifically, assuming a system with two levels of caches, we use Luo's model [Luo et al. 1998] that expresses CPI as:

$$
C P I=C P I_{L 2->\infty}+h_{m} \times t_{m},
$$

where $C P I_{L 2->\infty}$ indicates the CPI of the program when the lowest level onchip cache (e.g., the L2 cache) has infinite size, $h_{m}$ and $t_{m}$ indicate the number of L2 misses per instruction and the average penalty (in number of cycles) of an L2 miss respectively. With resource stealing, we attempt to steal L2 cache capacity from an Elastic(X) job and reallocate it to Opportunistic jobs. The effect of reduced L2 cache size for the Elastic(X) job is a higher L2 cache miss rate (hence higher $h_{m}$ ), but other variables would remain roughly unchanged. ${ }^{2}$ Since $h_{m} \times t_{m}$ is only one component of the $C P I$, and all other components have positive (at least nonnegative) values, an increase of $\mathrm{X}$ in $h_{m}$ would result in a less than X increase in CPI. We exploit this observation to guide our resource stealing algorithm such that it removes cache capacity from an Elastic $(\mathrm{X})$ job

\footnotetext{
${ }^{2}$ The CPI of infinite L2 cache $\left(C P I_{L 2->\infty}\right)$ is mostly affected by physical L1 and L2 cache organization, and not affected much by L2 cache partition sizes. The average latency of an L2 miss $\left(t_{m}\right)$ may be affected as memory bus contention increases due to the increase in the number of L2 cache misses. However, this can be mitigated by prioritizing memory requests from Elastic(X) jobs over those from Opportunistic jobs. In addition, we can monitor bus utilization and disable resource stealing when the utilization is higher than a certain threshold beyond which queuing delay for memory requests is no longer minor.
}

ACM Transactions on Architecture and Code Optimization, Vol. 7, No. 3, Article 14, Pub. date: December 2010. 


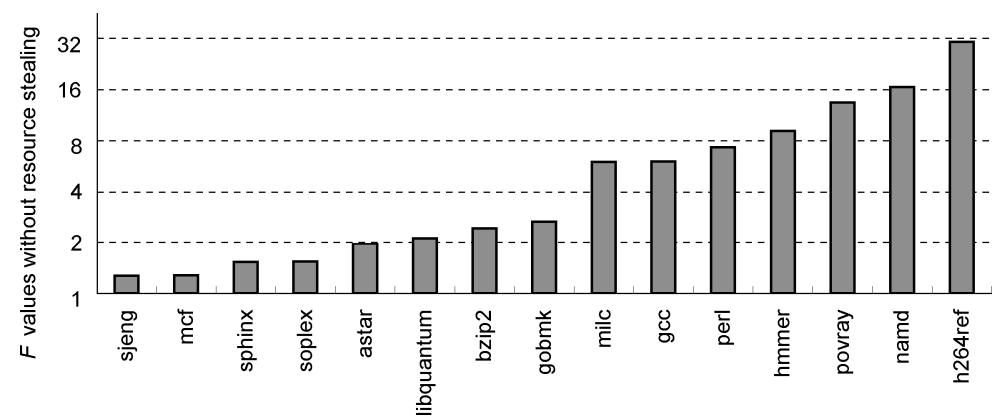

Fig. 4. The $\mathcal{F}$ (i.e. $\frac{C P I}{h_{m} \times t_{m}}$ ) values of fifteen SPEC2006 benchmarks. Detailed simulation parameters are described in Section 7 .

but without increasing the job's L2 cache miss rate by more than X. We refer to this algorithm as $R S_{\text {base }}$.

Note that our criteria of allowing the L2 cache miss rate to increase by not more than $\mathrm{X}$ is likely to be conservative, we will address this in the next several sections. Finally, monitoring the L2 miss rate increase is achievable with relatively simple hardware modification which will be described in Section 4.6.

\subsection{The Conservativeness of the $R S_{\text {base }}$ Algorithm}

In $R S_{\text {base }}$, the criteria of allowing the L2 cache miss rate to increase by not more than X, while fairly simple, is sometimes too conservative.

Let $C P I$ and $h_{m}$ denote the original CPI and the number of L2 misses per instruction, while $C P I^{\prime}$ and $h_{m}^{\prime}$ denote the CPI and the number of L2 misses per instruction when resource stealing is applied. Since both $C P I_{L 2->\infty}$ and $t_{m}$ can be assumed to be unaffected by resource stealing, the relative increase in CPI, denoted as $\triangle C P I$, can be expressed by Equation (2). Since the relative increase in miss rate $(\triangle M i s s$ Rate $)$ is equal to $\frac{h_{m}^{\prime}-h_{m}}{h_{m}}$, we can express $\triangle C P I$ in terms of $\triangle$ MissRate, as shown in Equation (3).

$$
\begin{gathered}
\triangle C P I=\frac{C P I^{\prime}-C P I}{C P I}=\frac{\left(h_{m}^{\prime}-h_{m}\right) \times t_{m}}{C P I} \\
\triangle C P I=\frac{h_{m}^{\prime}-h_{m}}{h_{m}} \times \frac{h_{m} \times t_{m}}{C P I}=\triangle \text { MissRate } \times \frac{1}{\mathcal{F}} .
\end{gathered}
$$

Equation (3) denotes $\frac{C P I}{h_{m} \times t_{m}}$ as $\mathcal{F}$. The equation implies that in order to ensure that CPI does not increase by more than X, the increase in miss rate should be less than $X \times \mathcal{F}$. Note, however, that $\mathcal{F} \geq 1$ because $h_{m} \times t_{m}$ is only one component of $C P I$. The $R S_{\text {base }}$ algorithm conservatively assumes that $\mathcal{F}=1$, hence the maximum allowable increase in the relative miss rate is sometimes too small, that is, too conservative, because in practice, $\mathcal{F}$ is often much larger than one. Figure 4 presents the $\mathcal{F}$ values for fifteen SPEC2006 benchmarks (resource stealing is not applied). While $\mathcal{F}$ varies between 1.26 and 30.1 for different benchmarks, for two thirds of the benchmarks, the value is larger than 2 , indicating that to tolerate a slowdown of $\mathrm{X}$, the miss rate can be allowed to increase by more than $2 \times \mathrm{X}$. Therefore, assuming $\mathcal{F}=1$ as in $R S_{\text {base }}$ is 
too conservative, preventing resource stealing from reallocating all the excess cache capacity from Elastic(X) jobs to Opportunistic jobs.

In order to recover more excess cache capacity from Elastic $(X)$ jobs, we need to estimate a more accurate value of $\mathcal{F}$, rather than assuming its lowest value of 1. This requires us to know the original (i.e., without resource stealing) values of the components that make up $\mathcal{F}: C P I, h_{m}$, and $t_{m}$. The original value of $h_{m}$ can be directly measured through additional duplicate tags which maintain the tags that are unaffected by resource stealing (the details of using duplicate tags are discussed in Section 4.6). The original value of $t_{m}$ is unknown, but we can replace it with its upper bound, which is the maximum miss penalty suffered when instruction level parallelism (ILP) and memory level parallelism (MLP) do not hide any part of it (i.e. the full memory latency, denoted as $T_{m}$ ). Therefore, the remaining challenge is to know what the original value of $C P I$. Unfortunately, once resource stealing is applied, the CPI is affected and its original value is no longer measurable. Thus, we can only rely on estimating it using statistical methods.

\subsection{CPI Estimation Using Sampling}

To approximate the original value of CPI, we choose to use a sampling technique. An application's execution is divided into intervals with equal number of instructions. We choose one sampling interval to measure CPI with no resource stealing applied, followed by $\alpha$ consecutive intervals in which resource stealing is applied, where $\alpha$ is an adjustable parameter. Hence, resource stealing is only applied for $\frac{\alpha}{1+\alpha}$ of the intervals. We collect the CPIs from past sampling intervals, and take their average $(\overline{C P I})$. Assuming that the CPI in the next set of nonsampling intervals have the same value as $\overline{C P I}$, and adjusting for the fact that there is no increase in miss rate during sampling intervals, we can express the new resource stealing criteria as:

$$
\triangle \text { MissRate } \leq X \times \frac{\overline{C P I}}{h_{m} \times T_{m}} \times \frac{\alpha+1}{\alpha},
$$

where $\mathrm{X}$ is the maximum allowable slowdown. We refer to this resource stealing

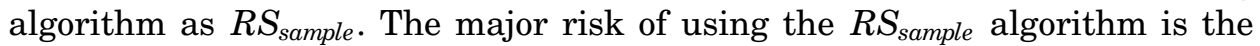
accuracy of estimating the original CPI using the average of sampled CPIs $(\overline{C P I})$. Sampling is imperfect. $\overline{C P I}$ collected through sampling may overestimate or underestimate the original CPI values in future intervals where resource stealing is applied. When $\overline{C P I}$ underestimates future original CPIs, no harm is done because it puts a lower cap on the increase in miss rate than what is allowed in reality. However, when $\overline{C P I}$ overestimates future original CPIs, the increase in miss rate may become higher than what is allowed and overshoot the maximum slowdown in CPI. To avoid overshooting slowdown, we add a safety margin to the estimated $\overline{C P I}$ using a sound statistical method.

\subsection{CPI Sampling with Safety Margin}

To achieve that, we need to measure the degree of confidence of the $\overline{C P I}$ in estimating the future original CPIs, by relying on the assumption commonly 
used in statistical analysis that sample points are independent and identically distributed. However, it is well known that applications go through phases of execution in which one phase often exhibits a very different CPI compared to other phases. Hence, it is likely that sampled CPIs are identically distributed only over a shorter time period (within a phase) rather than over a longer time period (across phases). Therefore, we define a safe sample window, denoted by $W$, as the number of the most recently collected samples that are used in computing the degree of confidence. We then assume that CPI samples over $W$ are independent and identically distributed with a mean of $\mu$ and variance of $\sigma^{2}$. Based on these assumptions, the Central Limit Theorem implies that $\overline{C P I}$ over $W$ follows a normal distribution with a mean of $\mu$ and variance of $\sigma_{\text {sample }}^{2}=\frac{\sigma^{2}}{W}$. Hence, there is a $95 \%$ probability that the original CPI is larger than $\overline{C P I}-1.64 \times \sigma_{\text {sample }}$. Similarly, there is a $99 \%$ probability that it is larger than $\overline{C P I}-2.33 \times \sigma_{\text {sample }}$. As a result, $\overline{C P I}-1.64 \times \sigma_{\text {sample }}$ forms the lower bound of the original CPI value with $95 \%$ confidence. We denote this estimated lower bound as $C P I_{\min }$, and our criteria for miss rate increase becomes:

$$
\triangle \text { MissRate } \leq X \times \frac{C P I_{\min }}{h_{m} \times T_{m}} \times \frac{\alpha+1}{\alpha} .
$$

We refer to this modified algorithm as $R S_{\text {safe }}$. Compared with $R S_{\text {sample }}, R S_{\text {safe }}$ is relatively conservative in stealing resource since $C P I_{\min }$ includes a margin of safety that is often lower than the actual CPI. During a period in which sampled CPI are volatile (i.e. their values fluctuate wildly), the estimated standard deviation becomes high, $C P I_{\min }$ becomes small, lowering the cap on miss rate increase. However, when sampled CPI are relatively uniform, the estimated standard deviation becomes low, $C P I_{\min }$ becomes large, and the cap on miss rate increase is allowed to increase significantly. Hence, resource stealing in $R S_{\text {safe }}$ tends to be more aggressive when stable phases are detected, and becomes conservative when unstable phases or transitions between phases are encountered, making $R S_{\text {safe }}$ more robust compared to $R S_{\text {sample }}$. Empirically, we found that in some cases, the computed $\mathcal{F}$ drops below one, which is unnecessarily too conservative. In such cases, we simply revert the resource stealing criteria to the base scheme $\left(R S_{\text {base }}\right)$ temporarily.

The sample safe size $W$ obviously impacts the effectiveness of the $R S_{\text {safe }}$ algorithm. Ideally, $W$ must be adjusted such that it is able to detect most of program phases with stable CPI values. If $W$ is too large, it may include multiple phases with highly different CPI values, and the algorithm becomes overly conservative and has less opportunity to perform resource stealing. However, if $W$ is too small, the accuracy of average and variance of sampled CPIs is reduced. In our experiments, we found a safe sample window of three intervals to deliver reasonable accuracy and coverage for resource stealing.

\subsection{Microarchitecture Support}

The resource stealing technique requires monitoring the miss rate increase due to partition changes, hence we need a mechanism to dynamically obtain the miss rates for both the reduced and original partition cases. To achieve this, we 
use a straightforward method that utilizes an additional duplicate cache tag array [Qureshi et al. 2006; Subramanian et al. 2006] which keeps track of what blocks the cache would have if resource stealing had not been applied, while the main cache tag array keeps track of the actual cache content. To reduce the storage overhead of keeping the duplicate tag array, we also employ set sampling [Qureshi et al. 2006; Qureshi and Patt 2006], in which only a few sets are augmented with duplicate tags and profiled to infer the global cache behavior. We let the stream of all L2 cache accesses be visible to both tag arrays so that only their numbers of misses differ.

When an Elastic $(\mathrm{X})$ job runs, the resource stealing technique is activated. For $R S_{\text {sample }}$ and $R S_{\text {safe }}$ algorithms, the CPI sampling technique is applied as well. At the beginning, the Elastic(X) job's partition size is reduced by one way. This "stolen" way is reallocated to one of the Opportunistic jobs. The target allocation counters for the Elastic $(\mathrm{X})$ job and the Opportunistic job are updated, which allows the cache to converge to the new partitions over time. In the meantime, the duplicate tags keep track of the total number of misses for the Elastic $(\mathrm{X})$ job without resource stealing. Based on the running resource stealing algorithm, if the extra number of misses in the main tags reaches or exceeds the corresponding threshold (Equations (4), (5) compared to that in the duplicate tags, resource stealing is canceled and all the stolen ways are returned to the Elastic $(\mathrm{X})$ job. Otherwise, in the next time interval we steal another way from the Elastic(X) job. Note that while repartitioning occurs at periodic intervals, the number of misses in the duplicate tag array and main tag array are not reset at each interval. This ensures that the total number of misses since the start of the Elastic $(\mathrm{X})$ job does not increase by more than the threshold.

\section{DYNAMIC RESOURCE STEALING MECHANISM}

In Section 4, we have discussed three different algorithms that detect the excess cache capacity from Elastic(X) jobs. However, recovery of excess cache capacity from Elastic(X) jobs alone cannot ensure better overall throughput if Opportunistic jobs cannot improve their throughput by more than the loss of throughput in Elastic(X) jobs. Ideally, resource stealing should be dynamically activated and deactivated depending on whether overall throughput improves. In this section, we present such a mechanism that we refer to as $R S_{d y n}$.

One way to monitor whether resource stealing is beneficial or not is to monitor the total number of L2 cache misses suffered by the system. If after applying resource stealing the total number of misses fails to decrease by a desired amount, it is likely that resource stealing has not been beneficial, since the reduction in number of misses in Opportunistic jobs cannot compensate for the increased number of misses in Elastic(X) jobs. More specifically, we demand that resource stealing, which may cause a slowdown of Elastic $(\mathrm{X})$ jobs by up to $\mathrm{X} \%$, compensates for the slowdown by reducing the total number of misses by more than $\mathrm{X} \%$. If this condition is not satisfied, we deactivate resource stealing.

In order to monitor the number of misses for both cases (with vs. without resource stealing) simultaneously, we use the duplicate tags in a novel way, 
which now serves two purposes: (1) enforcing resource stealing criteria for Elastic(X) jobs, and (2) monitoring whether resource stealing has been beneficial. To achieve the latter purpose, we keep two counters $\left(C_{R S}\right.$ and $\left.C_{N o R S}\right)$ that record the number of cache misses with and without resource stealing, respectively.

At the end of each repartitioning interval, we obtain the miss counters from both the main and duplicate cache tags. If resource stealing was applied in the just-completed interval, the number of misses from the main tag is added to $C_{R S}$, while the number of misses from the duplicate tag is added to $C_{N o R S}$. If $\frac{C_{R S}}{C_{N o R S}}>1-X$, indicating the reduction of number of misses under resource stealing to be less than X\%, resource stealing is deactivated in the next interval. Otherwise, resource stealing remains active in the next interval.

If resource stealing was not applied, that is, deactivated, in the justcompleted interval, the number of misses from the main tag reflects that of without resource stealing, so it is added to $C_{N o R S}$. Simultaneously, rather than turning off the resource stealing algorithm completely, we apply it to the duplicate tags so that we can still measure the number of misses due to resource stealing simultaneously without affecting cache performance. The number of misses obtained in the duplicate tags is then added to $C_{R S}$. If $\frac{C_{R S}}{C_{N o R S}} \leq 1-X$, resource stealing is activated in the next interval. Otherwise, it remains deactivated in the next interval.

Note that $R S_{d y n}$ can be used in combination with $R S_{\text {base }}, R S_{\text {sample }}$, and $R S_{\text {safe }}$ because it only decides whether to activate or deactivate resource stealing in the next interval. The basic algorithms of $R S_{\text {base }}, R S_{\text {sample }}$, and $R S_{\text {safe }}$ continue to work on the main cache tag (if resource stealing is active) or the duplicate cache tags (if resource stealing is deactivated). The number of misses collected at the point of transition between resource stealing activation/deactivation is slightly distorted because of the different cache states at the main vs. duplicate tags. However, in our experiments, we found that such transitions are rare because of the stable nature of whether resource stealing is beneficial or not, hence the effect of the distortion is small. In addition, in the cases where transitions occur frequently, the distortion can be largely mitigated by increasing the size of the intervals.

\section{LOCAL ADMISSION CONTROLLER IMPLEMENTATION}

For the Local Admission Controller (LAC), we implement a First Come, First Served (FCFS) scheduling algorithm using a basic resource allocation model from Rajkumar et al. [1997]. The LAC maintains a list of vectors that encode processor core and cache capacity resources and the timeslots in which they are available. A job specifies its QoS target through a resource request vector which encodes the amount of resources needed for each resource type, and for how long (based on the maximum wall-clock time) they are needed. For a Strict or Elastic(X) job, The LAC tries to find the earliest timeslot in which this vector can be fit before the job's deadline. If such a timeslot is found, the job is accepted and the resources are reserved in that timeslot. An Opportunistic job 
is always accepted if there are spare resources not already taken up by Strict or Elastic(X) jobs.

To keep the OS thread scheduler unchanged, we implement the LAC as a user-level program. The LAC has a scheduler queue to store accepted jobs and manage their resource and timeslot reservations. Once it is time to start a job, the job is submitted to the OS. If the job is an Elastic(X) job, resource stealing is also activated. To avoid timesharing from violating a Strict or Elastic(X) job's deadline, the LAC pins only one such job to one processor core. However, the LAC may pin multiple Opportunistic jobs on a core that is not already assigned to Strict or Elastic(X) jobs.

\section{EVALUATION METHODOLOGY}

Simulation Environment. To evaluate the proposed QoS framework, we use a full-system simulator based on Simics [Magnusson et al. 2002] to model a 4-core CMP node with Fedora Core 4 Linux as the operating system. Each core is an in-order processor with a $2 \mathrm{GHz}$ clock frequency. Each core has a private L1 instruction and a private $\mathrm{L} 1$ data cache with a $32 \mathrm{~KB}$ size, 4 -way associativity, 64-byte block size, LRU replacement policy, write back policy, and 2-cycle access time. The L2 cache is shared by all four cores, and is a unified cache with a $2 \mathrm{MB}$ size, 16-way associativity, 64-byte block size, modified LRU replacement policy (Section 4.1), write-back policy, and 10-cycle access time. The main memory is $4 \mathrm{~GB}$ in size with a 300 -cycle access time. The peak bandwidth to the main memory is $6.4 \mathrm{~GB} / \mathrm{s}$. The simulator ignores the impact of page mapping by assuming each job is allocated contiguous physical memory.

Resource Stealing. We employ set sampling to implement the duplicate tags used in resource stealing. The duplicate tags only cover $\frac{1}{8}$ of the total number of sets, which is conservative compared to 32 sets used in Qureshi and Patt [2006]. Every $8^{\text {th }}$ set is sampled. The interval for triggering cache repartitioning is 2 million instructions of the Elastic $(\mathrm{X})$ job.

To evaluate the $R S_{\text {sample }}$ and $R S_{\text {safe }}$ algorithms, the sampling parameter $\alpha$ is set to two for both $R S_{\text {sample }}$ and $R S_{\text {safe }}$ algorithms, which means that $33 \%$ of the overall intervals are spent on sampling and the rest for resource stealing. $\alpha$ is chosen to be small in order to achieve a high estimation accuracy. In each sampling interval, the first one million instructions are excluded from sampling as the cache is adjusting to the new partition size. The CPI of the next one million instructions is used to form a sample point. For the $R S_{\text {safe }}$ algorithm, the degree of confidence is $95 \%$ is used, and the sample safe size of three is used.

Individual Job. To evaluate our proposed QoS execution modes and resource stealing technique, we choose fifteen C/C++ benchmarks from SPEC2006 benchmark suite [Standard Performance Evaluation Corporation 2006]: gcc, bzip2, perl, gobmk, mcf, hmmer, sjeng, libquantum, h264ref, milc, astar, namd, soplex, povray, sphinx. We use the ref input sets for all benchmarks except for milc and soplex which use the train input sets because their ref input sets require too much memory. For each benchmark, we first inspect its source code 


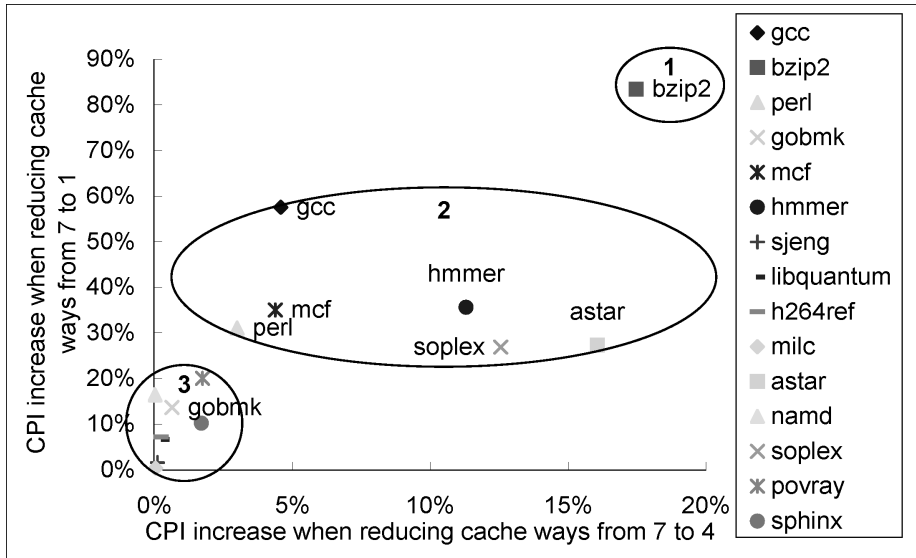

Fig. 5. The sensitivity of each benchmark to cache capacity.

Table I. The Benchmarks Used as Individual Jobs in the Evaluation

\begin{tabular}{|l|c|c|c|c|}
\hline Benchmark & Input Set & $\begin{array}{c}\text { L2 Miss } \\
\text { Rate }\end{array}$ & $\begin{array}{c}\text { L2 Misses } \\
\text { Per } \\
\text { Instruction }\end{array}$ & $\begin{array}{c}\text { Number of } \\
\text { Skipped } \\
\text { Instructions }\end{array}$ \\
\hline bzip2 & ref.chicken & $20 \%$ & 0.0055 & $315 \mathrm{M}$ \\
\hline hmmer & ref.retro & $17 \%$ & 0.001 & $0.3 \mathrm{M}$ \\
\hline gobmk & ref.nngs & $24 \%$ & 0.004 & $267 \mathrm{M}$ \\
\hline
\end{tabular}

and identify its initialization routines. Then we skip the initialization routines and simulate the next 200 million instructions.

To reduce the number of benchmarks that we need to evaluate, we classify the benchmarks according to their cache space sensitivity. For each benchmark, we calculate the CPI increase when we reduce its L2 cache allocation from 7 ways to 1 way, and from 7 ways to 4 ways. Then we plot them in a twodimensional space (Figure 5).

From the figure, we can roughly classify the fifteen benchmarks into three groups based on how sensitive they are to the allocated cache space: highly sensitive (Group 1), moderately sensitive (Group 2), and insensitive (Group 3). Similar classification methods can be found in Chang and Sohi [2007] and Qureshi and Patt [2006]. Highly sensitive benchmarks are ideal beneficiaries of resource stealing, whereas insensitive benchmarks are ideal donors for resource stealing. From each group, we choose one representative benchmark: bzip2 from Group 1, hmmer from Group 2 and gobmk from Group 3. The L2 miss rates and L2 misses per instruction of these benchmarks when they are allocated 7 cache ways are listed in Table I.

Workload Composition. We construct 10-job workloads and measure the wall clock time to complete all ten jobs. Each job requests a processor core and L2 cache capacity of $896 \mathrm{~KB}$ ( 7 ways in the 16 -way L2 cache). We assume incoming jobs with Poisson arrival, with an inter-arrival time that assumes full computation capacity utilization of a 128-CMP server. Specifically, on a 
Table II. Execution Modes Configurations

\begin{tabular}{|l|c|}
\hline Configuration & Percentage of Jobs in Various Execution Modes \\
\hline All-Strict & $100 \%$ Strict \\
\hline Hybrid-1 & $70 \%$ Strict $+30 \%$ Opportunistic \\
\hline Hybrid-2 & $\begin{array}{c}40 \% \text { Strict }+30 \% \text { Elastic(5\%) + } \\
30 \% \text { Opportunistic }\end{array}$ \\
\hline All-Strict + & $\begin{array}{c}100 \% \text { Strict, jobs with moderate or } \\
\text { relaxed deadlines are automatically downgraded. }\end{array}$ \\
\hline EqualoDown & $\begin{array}{c}\text { No admission control, default Linux job scheduling, } \\
\text { L2 cache is equally partitioned among cores. }\end{array}$ \\
\hline
\end{tabular}

Table III. Mixed-Benchmark Workloads

\begin{tabular}{|l|c|}
\hline Type & Workload Composition \\
\hline Mix-1 & $\begin{array}{c}\text { hmmer (Strict), gobmk (Elastic(5\%)) and } \\
\text { bzip2 (Opportunistic) }\end{array}$ \\
\hline Mix-2 & $\begin{array}{c}\text { hmmer (Strict), bzip2 (Elastic(5\%)) and } \\
\text { gobmk (Opportunistic) }\end{array}$ \\
\hline
\end{tabular}

4-core CMP, in one job's wall-clock time, there are on average $4 \times 128$ new jobs that arrive and probe the CMP's Local Admission Controller. Job deadlines are assigned as follows. We pseudo-randomly set $50 \%$ of them with a tight deadline $(t d-t a=1.05 \times t w), 30 \%$ with a moderate deadline $(t d-t a=2 \times t w)$ and $20 \%$ with a relaxed deadline $(t d-t a=3 \times t w)$.

To evaluate the various execution modes, we use five configurations shown in Table II. The base configuration is All-Strict. The EqualPart configuration mimics the Virtual Private Cache [Nesbit et al. 2007] by equally partitioning the cache capacity among all cores, but without an admission controller and bandwidth partitioning. In the Hybrid-2 configuration, unless otherwise specified, we employ $R S_{\text {safe }}$ algorithm and a slack value of $5 \%$ for each Elastic(X) job.

The 10-job workloads are constructed in two ways. First, we use instances of the same benchmark, that is, 10 instances of bzip2, 10 instances of $\mathrm{hmmer}$, and 10 instances of gobmk. In addition, we also construct two mixed-benchmark workloads shown in Table III. Mix-1 is a favorable workload for our framework since cache-sensitive bzip2 is the recipient of resource stealing, while cacheinsensitive gobmk is the donor of resource stealing. In contrast, Mix-2 is not favorable to our resource stealing technique.

\section{EVALUATION RESULTS}

In this section, we will present our experiment results, including the impact of various execution modes (Section 8.1), the impact of automatic mode downgrade (Section 8.2), evaluation of mixed workloads (Section 8.3), a selection of a good slack value (Section 8.4), evaluation of the resource stealing techniques (Section 8.5), the impact of $R S_{d y n}$ mechanism on overall throughput (Section 8.6) and characterization of the Local Admission Controller (Section 8.7). 


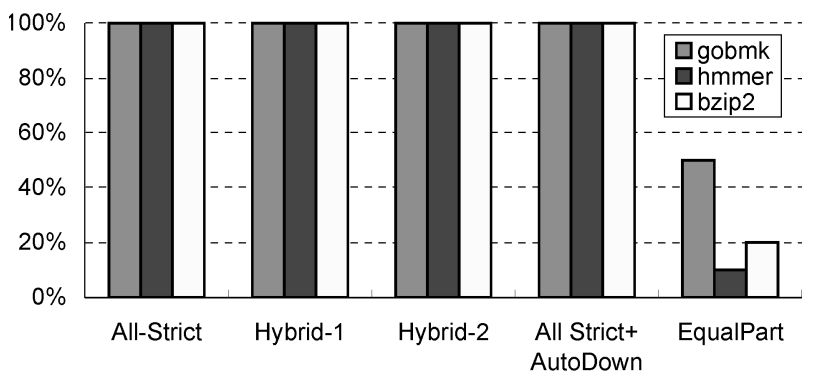

(a) Deadline hit rates for Strict and Elastic(X) jobs

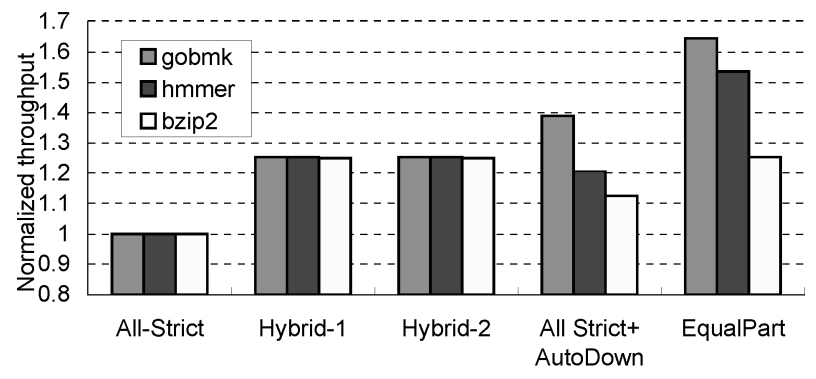

(b) Job throughput

Fig. 6. Comparing QoS and throughput of different configurations.

\subsection{Impact of Various Execution Modes}

Let deadline hit rate refer to the fraction of jobs that meet their deadlines. Figure 6(a) shows the deadline hit rates for various configurations (Table II) with workloads consisting of ten identical instances of a single benchmark. For our QoS framework, the deadline hit rate is only computed for Strict and Elastic $(\mathrm{X})$ jobs. The figure shows a consistent result of $100 \%$ deadline hit rate in our QoS framework. In contrast, the deadline hit rates are only 50\%, 10\% and $20 \%$ in EqualPart for gobmk, hmmer and bzip2, respectively. This is because in EqualPart, the lack of an admission controller causes jobs to be accepted continuously despite the fact that the CMP no longer has sufficient computation capacity to meet the jobs' deadlines. This observation reinforces the argument that partitioning the cache capacity among cores alone cannot fully provide QoS. Only after incorporating an admission control policy and using appropriate QoS targets can jobs reliably meet their deadlines.

Figure 6(b) compares the job throughput of the single-benchmark workloads on various configurations, measured as the total wall-clock time to complete the first ten accepted jobs. The throughput is normalized to the All-Strict case. Comparing All-Strict and EqualPart, the figure clearly shows that in all workloads, providing strict QoS comes at a cost of significantly lower job throughput, e.g. the throughput in EqualPart is higher by $64 \%, 54 \%$ and $25 \%$ for gobmk, hmmer and bzip2, respectively. There are several factors that cause this result. The first is various external resource fragmentation such as the processor cores (only two jobs run simultaneously, leaving two idle cores) and cache capacity (only 14 ways in the 16-way L2 cache are allocated). The second is the 
internal cache capacity fragmentation if the jobs do not fully utilize their allocated cache partitions, which is especially the case for cache-insensitive benchmarks such as gobmk. In contrast, the EqualPart configuration does not suffer from any external resource fragmentation and suffers little from internal resource fragmentation. As a result, the more cache sensitive the jobs in a workload are, the smaller throughput reduction the workload suffers from when QoS is provided.

In Hybrid-1, the existence of Opportunistic jobs effectively removes external core and cache capacity fragmentation, resulting in a significant $25 \%$ improvement in throughput for all workloads. However, since internal cache capacity fragmentation still exists (especially in gobmk and hmmer), the throughput is still lower than that in EqualPart. In Hybrid-2, the existence of Elastic(X) jobs conceptually allows some cache capacity to be stolen and reallocated to Opportunistic jobs, reducing some internal cache capacity fragmentation. However, the figure shows that the throughput of all the workloads in Hybrid-2 are almost the same as those in Hybrid-1. Upon closer analysis, we find that indeed Opportunistic jobs complete sooner but the overall job throughput is largely unaffected because the tenth accepted job is a Strict job and it completes at almost the same time in Hybrid-1 and Hybrid-2 for all workloads. Note that internal cache capacity fragmentation still exists in Strict jobs (70\% in Hybrid-1 and $40 \%$ in Hybrid-2) and it cannot be removed without the risk of reducing throughput and missing deadlines. This prevents Hybrid-1 and Hybrid-2 from matching the throughput of EqualPart.

Finally, in All-Strict+AutoDown, automatic mode downgrade is applied to Strict jobs to remove some of the resource fragmentation, resulting in throughput improvements of 39\%, 20\% and 13\% for gobmk, hmmer and bzip2 respectively. Again, due to their higher internal cache capacity fragmentation, the throughput improvement for gobmk and hmmer are higher than that of bzip2. The throughput improvement over All-Strict is substantial, especially considering that the optimization does not require users to downgrade the modes of their jobs. However, we note that since we apply automatic mode downgrade only to jobs whose deadlines are relaxed or moderate (Table II), only half of the jobs benefit from automatic mode downgrade. We can expect that if more jobs have moderate or relaxed deadlines, the throughput will be closer to that of EqualPart.

Overall, we conclude that while providing QoS imposes a severe penalty on throughput, a significant part of the throughput can be recovered through several schemes: providing various execution modes to users, and/or employing automatic mode downgrade transparently.

To further analyze the impact of various execution modes on wall-clock time, Figure 7 shows the average wall-clock time of jobs in different configurations for the single-benchmark workload of bzip2. The candle on each bar shows the range between the minimum and the maximum wall-clock time. The figure shows that with our QoS framework, Strict jobs in all configurations except All Strict+AutoDown have short and almost-constant wall-clock times. The Elastic(X) jobs in Hybrid-2 run slightly longer than Strict jobs because of resource stealing. However, their wall-clock time is still absent of much variation. As 


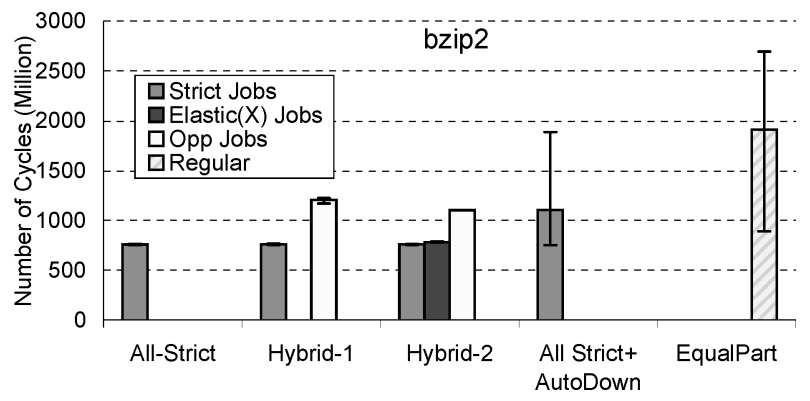

Fig. 7. The average and variation of wall-clock time for different configurations. The jobs are instances of bzip2.

expected, because resources are not reserved, Opportunistic jobs in Hybrid-1 and Hybrid-2 have a higher average and variation of wall-clock time compared to Strict jobs. Opportunistic jobs in Hybrid-2 have lower wall-clock time compared to those in Hybrid-1, thanks to the extra cache capacity stolen from Elastic $(\mathrm{X})$ jobs. We can see the impact of automatic mode downgrade on Strict jobs' wall clock time in the All-Strict+AutoDown configuration. Both the wall-clock time and variation in wall-clock time increase significantly compared to the Strict jobs in the All-Strict configuration. However, as long as the wall-clock time variation can be tolerated by the job, this is a good trade-off because all the jobs in the All-Strict+AutoDown still meet their deadlines and overall job throughput is significantly improved compared to the All-Strict case. Finally, the EqualPart configuration suffers from a high average and variation of wall-clock time, which is caused by the lack of admission control and resource reservation, as well as timesharing.

\subsection{Impact of Automatic Mode Downgrade}

To better understand how automatic mode downgrade improves throughput, we show the detailed execution of each job in the All-Strict and AllStrict+AutoDown for the single-benchmark workload of bzip2 in Figure 8. The $\mathrm{x}$-axes shows time in the number of millions of cycles, while the $\mathrm{y}$-axes shows the first ten accepted jobs. Boxes with solid lines represent the time from a job starting its execution until the time it completes, while boxes with dashed lines represent the amount of time between the job's completion and its deadline. In addition, automatically downgraded jobs are shown in a darker color and the arrows point to the time when they are to be switched back to the Strict execution mode.

From the figure, we can see that in the All-Strict case, only two jobs can be accepted and run simultaneously, leading to a low admission rate and throughput. It takes 3,883M cycles to complete all ten jobs. In the All-Strict+AutoDown case, it only takes $3,451 \mathrm{M}$ cycles to complete ten jobs for several reasons. The first reason is that because Strict jobs running in the Opportunistic mode do not reserve resources, more jobs can be accepted and started earlier. For example, the third job is executed earlier because the first job runs in the Opportunistic 


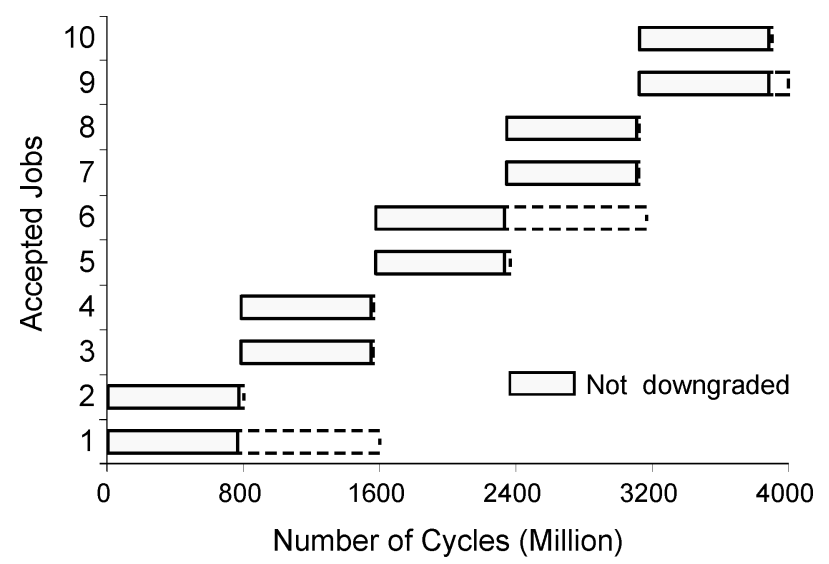

(a)

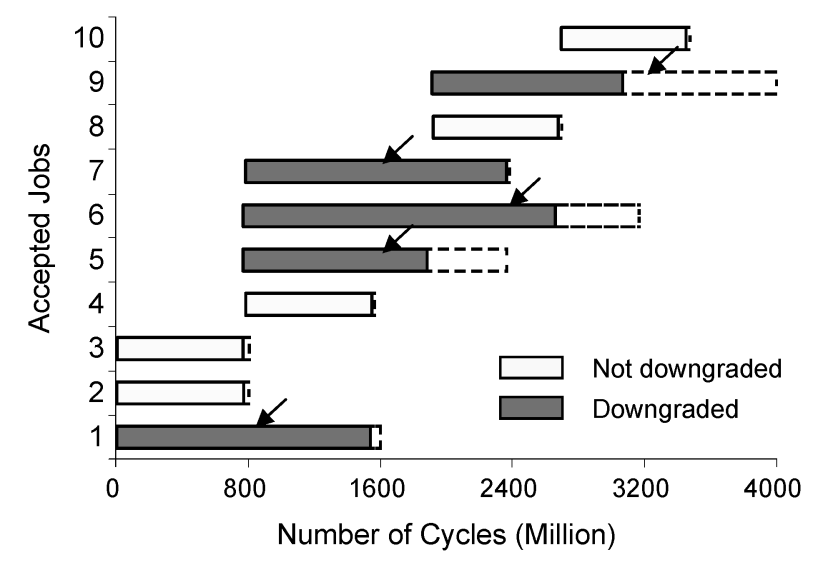

(b)

Fig. 8. Execution trace of ten accepted jobs in the All-Strict case (a) versus in the AllStrict+AutoDown case (b). The jobs are instances of bzip2.

mode rather than the Strict mode. The fifth, sixth, and seventh jobs are also admitted sooner and executed earlier. While automatically downgraded jobs run significantly slower, they utilize fragmented resources which otherwise would not have been utilized. The second reason is that when automatically downgraded jobs complete execution, the LAC reclaims their resources, allowing other jobs to be accepted earlier. For example, the completion of the fifth job allows the eighth job to be accepted and executed earlier, while the completion of the sixth job allows the tenth job to be accepted and executed earlier. Another observation is that out of the five automatically downgraded jobs, four of them (the first, fifth, sixth, and seventh) need to be switched back to the Strict mode because they do not complete early enough, while only one (the ninth job) is able to complete before it needs to be switched. Furthermore, the first and seventh jobs likely would not have met their deadlines had resources and timeslot not been reserved. This emphasizes the importance of reserving 


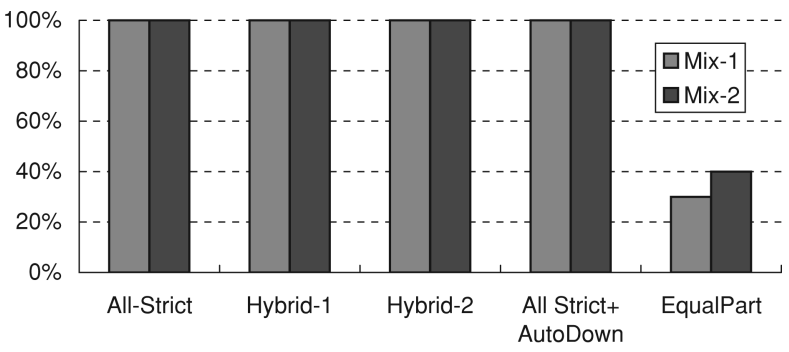

(a) Deadline hit rates for Strict and Elastic(X) jobs

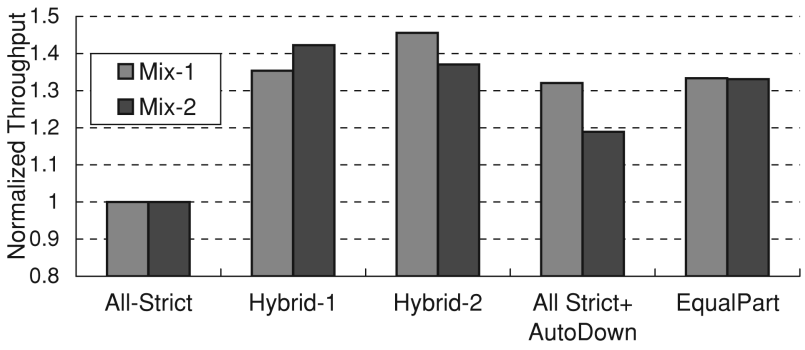

(b) Job throughput

Fig. 9. Comparing two mixed-benchmark workloads.

resources and timeslots for automatically downgraded jobs in order to guarantee meeting their deadlines.

\subsection{Evaluating Mixed-Benchmark Workloads}

So far we have only used single-benchmark workloads to evaluate our QoS framework. In this section, we evaluate two mixed-benchmark workloads described in Table III. Recall that Mix-1 represents an ideal workload for resource stealing: the cache-sensitive benchmark (bzip2) forms Opportunistic jobs while the cache-insensitive benchmark (gobmk) forms Elastic(5\%) jobs. Mix-2, on the other hand swaps the execution modes of bzip2 and gobmk, so it is not an ideal workload for resource stealing.

Figure 9(a) shows the deadline hit rates for different configurations. While our QoS framework achieves 100\% deadline hit rates for all Strict and Elastic(X) jobs in mixed-benchmark workloads, EqualPart has low deadline hit rates (30\% for Mix-1 and 40\% for Mix-2). Figure 9(b) shows the job throughput for Mix-1 and Mix-2 normalized to the respective All-Strict cases. Overall, all of Hybrid-1, Hybrid-2, and All-Strict+AutoDown achieve a significant improvement in throughput compared to All-Strict. The throughput improvements achieved in Hybrid-1 and Hybrid-2 sometimes even exceed that of EqualPart. This is a significant result considering that while a majority of jobs miss their deadlines in EqualPart, they meet their deadlines in our QoS framework while simultaneously achieving higher throughputs.

Comparing throughput improvement between the two workloads, Mix-1 achieves lower throughput improvement than Mix-2 in Hybrid-1 (35\% vs. 42\%) but higher than Mix-2 in Hybrid-2 (45\% vs. 37\%). This is because in Hybrid-1, 


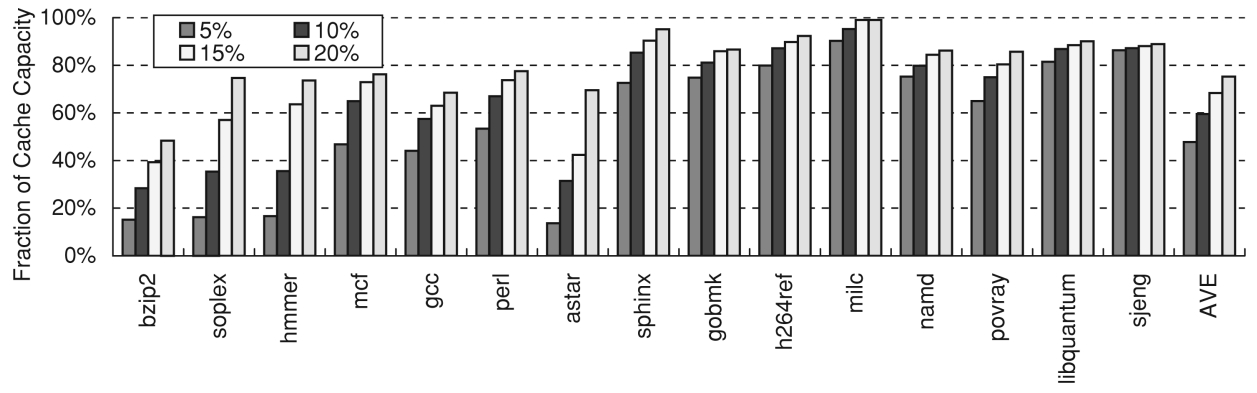

Fig. 10. The estimated fraction of cache capacity that can be stolen when the performance slack value varies from $5 \%$ to $20 \%$. The full evaluation setup can be found in Section 7 .

the Opportunistic jobs can only utilize unallocated cache capacity, and because bzip2 is more cache sensitive than gobmk, instances of bzip2 have more restricted throughput compared to instances of gobmk. However, in Hybrid-2, Opportunistic jobs benefit from extra cache capacity stolen from Elastic(X) jobs. In this case, Opportunistic bzip2 jobs benefit more from resource stealing than Opportunistic gobmk jobs, while at the same time Elastic(X) gobmk jobs can give up more of their cache space and are slowed down less than Elastic(X) bzip2 jobs. The outcome of this combination is that resource stealing is more effective for Mix-1 than for Mix-2. The consequence of the observation is that resource stealing should be applied selectively for different workloads. We will show how dynamic activation and deactivation of resource stealing addresses this problem in Section 8.6.

\subsection{Selection of a Good Slack Value}

One dilemma with the Elastic $(\mathrm{X})$ execution mode is with regard to the value selection of X: who should determine its value, and what value produces good performance? It may be too much to expect end users from choosing a good value of performance slack. Rather, the system should recommend a good value to end users. In this section, we attempt to address the dilemma.

Our first step is to find out the relationship between the amount of slack and the excess cache capacity that can be recovered from an application. Intuitively, we want to recover as much as excess cache capacity for as little slack as possible. To answer this question, we calculate the desired miss rate increase of each benchmark for the slack values varied from 5\% to 20\% by using Equation (3). The excess cache capacity that can be stolen to produce the desired increase in the cache miss rate is shown in Figure 10.

From Figure 10, we can see nonuniform behavior across benchmarks. For some benchmarks, the amount of excess cache capacity that can be recovered is low ( $<20 \%$ in bzip2, soplex, $h m m e r$ and astar for 5\% slowdown), because these benchmarks are highly cache sensitive. They belong to Group 1 or Group 2, as shown in Figure 5. For other benchmarks, more than $40 \%$ of the cache capacity can be stolen even for $5 \%$ slowdown. As the cap on slowdown increases, not much more cache capacity can be recovered, as much of the excess cache capacity has already been recovered with the 5\% slowdown. However, since 


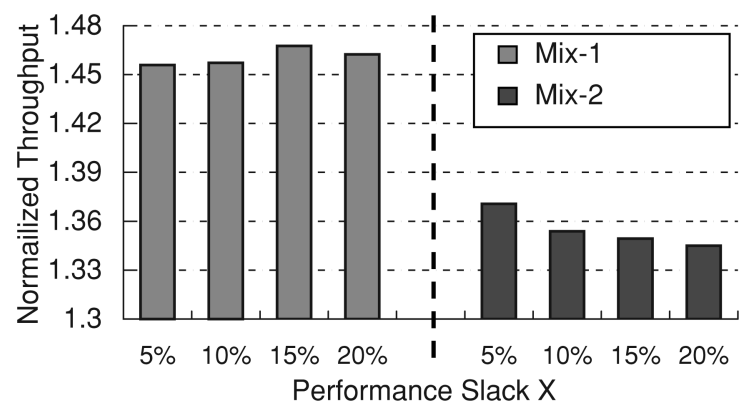

Fig. 11. Normalized throughput of Mix-1 and Mix-2 workload with different slack values when $R S_{\text {safe }}$ algorithm is used in Hybrid-2 configuration.

most benchmarks are much less cache sensitive than bzip2, soplex, hmmer, and astar, the average excess cache capacity that can be recovered shows a diminishing return along the increase in slowdown. On average, the stolen cache capacity will only increase from $48 \%$ to only $75 \%$ when the slack value increases from $5 \%$ to $20 \%$.

Figure 11 shows the overall system throughput and how it is affected by different slack values used for Elastic(X) jobs, for Mix-1 and Mix-2 workloads using the Hybrid-2 configuration. The throughput is normalized to the case in which all jobs run in strict mode (i.e., All-strict). The figure confirms that there is a lack of compelling evidence of the benefit of using a large slack value. In Mix-1, there is only a very slight throughput improvement when we use a larger slack value ( $47 \%$ with $15 \%$ vs. $46 \%$ with $5 \%$ ). The improvement occurs because even a small extra cache space received by the Opportunistic jobs (bzip2) can improve their throughput significantly, up to a point in which the improvement is offset by the slowdown in Elastic $(\mathrm{X})$ jobs. In Mix-2, a large slack is actually harmful because the Elastic $(\mathrm{X})$ jobs (bzip2) are highly cache space sensitive. Hence, they suffer a significant decline in throughput even when a small cache capacity is stolen from them. The Opportunistic jobs (gobmk) cannot benefit much from the extra cache capacity because they are cache space insensitive.

Overall, this result argues for a small slack value for several reasons. For most benchmarks, not much excess cache capacity can be recovered going from a $5 \%$ slowdown to a $20 \%$ slowdown. For a small number of benchmarks, while much excess cache capacity can be recovered going from a 5\% slowdown to a $20 \%$ slowdown, their performance is highly determined by the cache size they can access. Thus, stealing too much cache capacity from these benchmarks is risky as it can reduce their throughput significantly. Our experiments confirm that there is virtually no improvement in overall throughput from using a large slack value compared to using a small value of $5 \%$. In fact, in some cases, the overall throughput may decline with larger slack values. Hence, choosing a small $\mathrm{X}$ value of $5 \%$ offers a very attractive trade-off, and we can select it as a default for all Elastic jobs, unless users have reasons to override this setting. In the rest of the evaluation, we assume a 5\% slack unless stated otherwise. 


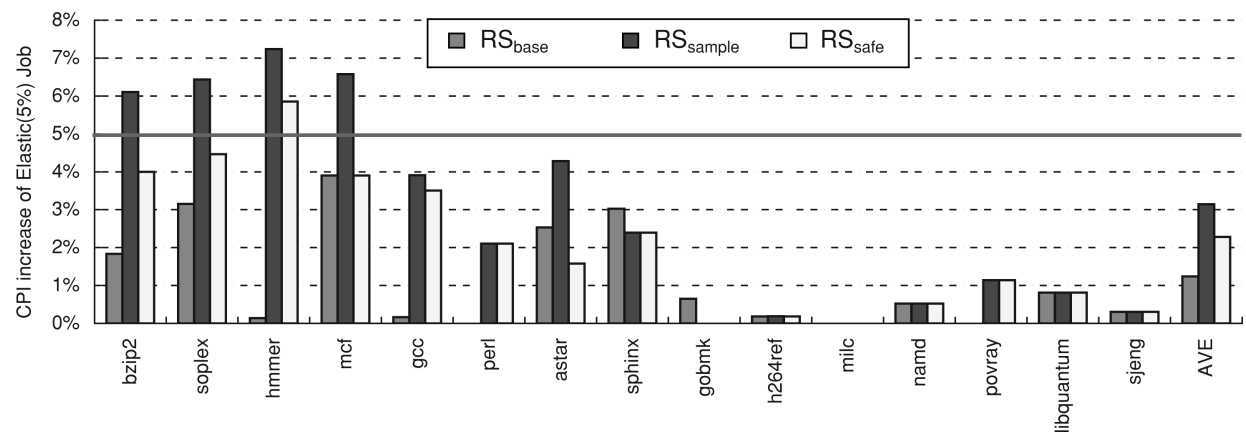

Fig. 12. The CPI increase of each benchmark when the amount of slack $\mathrm{X}$ is $5 \%$ with different resource stealing techniques.

\subsection{Resource Stealing Techniques Evaluation}

Figure 12 shows the CPI increase of various benchmarks when they run in the Elastic(5\%) mode with different resource stealing algorithms. From this figure, we can make several observations. First, the $R S_{\text {base }}$ algorithm is too conservative for almost all benchmarks. The actual CPI increase is between $1.8 \%$ to $3.8 \%$ for five benchmarks (bzip, soplex, mcf, astar and sphinx), and is much lower (less than 1\%) in ten other benchmarks.

Comparing $R S_{\text {sample }}$ to $R S_{\text {base }}$, it is clear that $R S_{\text {sample }}$ is a lot more aggressive in recovering excess cache capacity. It slows down half of the benchmarks by close to the allowed slowdown cap of 5\%. However, this aggressiveness causes the slowdown to overshoot the maximum slowdown for benchmarks $(6.1 \%, 7.2 \%, 6.4 \%, 6.7 \%$ for bzip2, soplex, hmmer and $m c f$ respectively). Frequent slowdown overshooting is not desirable even in systems with soft QoS requirements. The main reason for the overshooting is the $\overline{C P I}$ collected over sample intervals often overestimates the actual CPI when no resource stealing is applied.

Note that for cache space insensitive benchmarks (e.g., h264ref, milc, namd, libquantum, sjeng, etc.), the CPI increases are very low for all algorithms. This is because they have small working set, hence the miss rate of those benchmarks cannot be increased as expected no matter how many cache ways are stolen (each job is allocated a minimum of one cache way to avoid starvation). However, it does not mean that resource stealing is useless: a large excess cache capacity can actually be recovered using resource stealing (see Figure 10).

Comparing $R S_{\text {safe }}$ to $R S_{\text {sample }}$, for the four benchmarks that are highly cache sensitive (bzip2, soplex, $h m m e r$, and $m c f$ ), $R S_{\text {safe }}$ is much less aggressive than $R S_{\text {sample }}$, preventing slowdown overshooting in most cases except one benchmark (hmmer with the amount of overshoot of less than 1\%). The slowdown overshoot in hmmer is caused by the higher CPI values collected during sampling intervals versus during non-sampling period. An occasional overshoot is consistent with the use of a $95 \%$ confidence interval, since it implies that on average, in 1 out of 20 cases, the safety margin is not sufficient in preventing an overshoot. Hence, an occasional small overshoot confirms that $R S_{\text {safe }}$ works as 


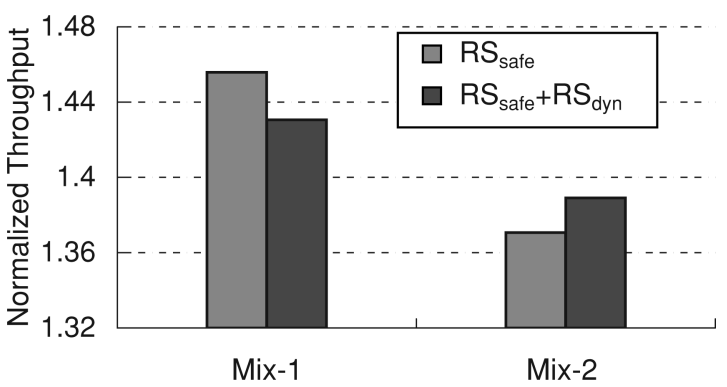

Fig. 13. Normalized throughput of Mix-1 and Mix-2 workload with $R S_{d y n}$ vs. without $R S_{d y n}$ mechanism in Hybrid-2 configuration. The $R S_{\text {safe }}$ algorithm is used for a slack X of $5 \%$.

intended. For the remaining eleven benchmarks that are less cache sensitive, it is almost as aggressive as $R S_{\text {sample }}$ that there is virtually small difference between them, except for astar.

In three cases, (astar, sphinx and gobmk), $R S_{\text {base }}$ slightly outperforms $R S_{\text {safe }}$ and/or even $R S_{\text {sample }}$. The reason is that in $R S_{\text {sample }}$ and $R S_{\text {safe }}$, only two thirds of the overall intervals are spent on resource stealing while $R S_{\text {base }}$ attempts to steal resource throughout the whole execution. While the miss rates are increased more by $R S_{\text {sample }}$ or $R S_{\text {safe }}$ when resource stealing is applied, only applying resource stealing for two thirds of all intervals cannot always compensate for the loss of opportunity for continuously stealing excess cache capacity. This effect can be largely mitigated by reducing the fraction of intervals used for sampling.

Overall, the proposed $R S_{\text {safe }}$ algorithm is much more effective in performing resource stealing compared to $R S_{\text {base }}$, while still preserving QoS of Elastic(X) jobs much better than $R S_{\text {sample }}$.

\subsection{Impact of $R S_{d y n}$ on Overall Throughput}

In previous section, we focus on evaluating the effectiveness of different resource stealing algorithms when they are activated throughout the entire execution. Figure 13 shows the overall throughput of Mix-1 and Mix-2 workload in Hybrid-2 configuration when $R S_{\text {safe }}$ is active all the time, versus when it is activated/deactivated dynamically using the $R S_{d y n}$ mechanism.

$R S_{d y n}$ mechanism affects the overall throughput of Mix-1 and Mix-2 differently. For Mix-1, when $R S_{d y n}$ is applied, the throughput improvement is lower than when $R S_{d y n}$ is not applied (43\% vs. $45 \%$ ). This is because $R S_{d y n}$ suspends the resource stealing in the first several repartitioning intervals until the reduction of total number of cache misses is larger than $5 \%$. During this period, bzip2 jobs cannot steal extra cache capacity. Hence, the loss of resource stealing opportunity hurts the performance of $R S_{d y n}$. However, this is a less serious problem in real-world environment because jobs run longer than in our evaluation. For Mix-2, resource stealing is harmful since the Opportunistic jobs gobmk (recipient of resource stealing) are cache space insensitive, while the Elastic(5\%) jobs bzip2 (donor of resource stealing) are cache space sensitive. 
Therefore, as expected, using $R S_{d y n}$ achieves higher throughput improvement (39\% vs. $37 \%$ ) because the resource stealing is deactivated during most of execution time. In general, $R S_{d y n}$ mechanism is useful in disabling resource stealing automatically when it is not likely to improve the overall throughput over the case in which resource stealing is not applied.

\subsection{Characterization of the Local Admission Controller}

In our framework, the LAC is implemented as a user level program and is fully simulated in our evaluation. The LAC incurs performance overheads when it performs admission tests and scheduling. However, since the LAC only performs a simple admission control policy and implements a simple scheduling algorithm, the occupancy of the LAC is less than $1 \%$ of each workload's wallclock time. If the number of jobs submitted to the CMP increases, or if the number of cores in the CMP increases, the LAC's overheads will increase proportionally although they likely remain low.

\section{CONCLUSIONS}

This article has presented a novel QoS framework that provides performance QoS through the use of appropriate QoS target specification and execution modes. It has also presented how throughput can be improved while still preserving QoS, by employing several techniques such as manual and automatic mode downgrade and resource stealing. Through this study, we discover several findings. First, QoS targets should be specified with Resource Usage Metrics (RUM) in order to fully provide QoS and to build an admission control policy. Second, QoS-enabling features such as the ability to dynamically partition caches and a resource manager which tries to meet the IPC target of all jobs, are by themselves insufficient for fully providing QoS. Third, substantial throughput is lost when we provide strict QoS with our framework due to external processor core and cache fragmentation, and internal cache fragmentation. Fourth, the two alternative QoS execution modes (Elastic(X) and Opportunistic) enable the system to recover much of the lost throughput by reducing resource fragmentation. Manual mode downgrade in general is more effective than automatic mode downgrade if there is an appropriate mixture of Elastic(X) and Opportunistic jobs. However, even when there are only Strict jobs, the overall throughput of the system can still be boosted by using automatic mode downgrade. Finally, resource stealing is a very useful microarchitecture technique for improving throughput by reallocating excess cache capacity from Elastic(X) jobs to Opportunistic jobs while still meeting the Elastic(X) jobs' QoS targets. Among the various resource stealing algorithms evaluated, $R S_{\text {safe }}$ achieves the best balance between improving throughput while preserving QoS. We found that a small slack value of $\mathrm{X}=5 \%$ is sufficient to recover most excess cache capacity, and the dynamic activation/deactivation of resource stealing mechanism can help ensure resource stealing always result in improved throughput. 


\section{REFERENCES}

Borkar, S., Dubey, P., Kahn, K., Kuck, D., Mulder, H., Pawlowski, S., And Rattner, J. $2005 . \quad$ Platform 2015: Intel processor and platform evolution for the next decade. Technology@Intel Mag.

BRANdEnBuRG, B. B. AND ANdERson, J. H. 2007. Integrating hard/soft real-time tasks and besteffort jobs on multiprocessors. In Proceedings of the 19th Euromicro Conference on Real-Time Systems. 61-70.

Buttazzo, G., Lipari, G., Caccamo, M., and Abeni, L. 2002. Elastic scheduling for flexible workload management. IEEE Trans. Comput. 51, 3, 289-302.

Chandra, D., Guo, F., KIM, S., And Solinin, Y. 2005. Predicting inter-thread cache contention on a chip multiprocessor architecture. In Proceedings of the 11th International Symposium on High Performance Computer Architecture. IEEE Computer Society, 340-351.

Chang, J. And SohI, G. S. 2007. Cooperative cache partitioning for chip multiprocessors. In Proceedings of the International Conference on Supercomputing. 242-252.

Сно, S. AND Jin, L. 2006. Managing distributed, shared L2 caches through OS-level page allocation. In Proceedings of the 39th Annual IEEE / ACM International Symposium on Microarchitecture. 455-468.

Емма, P. 1997. Understanding some simple processor-performance limits. IBM J. Resear. Develop. 41, 3.

Guo, F., Solinin, Y., Zhao, L., AND IyeR, R. 2007. A framework for providing quality of service in chip multi-processors. In Proceedings of the 40th Annual IEEE/ACM International Symposium on Microarchitecture. 343-355.

Hsu, L. R., Reinhardt, S. K., Iyer, R., And Makineni, S. 2006. Communist, utilitarian, and capitalist cache policies on CMPs: Caches as a shared resource. In Proceedings of the 15th International Conference on Parallel Architectures and Compilation Techniques. 13-22.

IYER, R. 2004. CQoS: A framework for enabling QoS in shared caches of CMP platforms. In Proceedings of the 18th Annual International Conference on Supercomputing. 257-266.

Iyer, R., Zhao, L., Guo, F., Solinin, Y., Markineni, S., Newell, D., Illikkal, R., Hsu, L., and Reinhardt, S. 2007. QoS policy and architecture for cache/memory in CMP platforms. In Proceedings of the ACM SIGMETRICS Conference on Measurement and Modeling of Computer Systems. $25-36$.

KIM, S., Chandra, D., AND Solihin, Y. 2004. Fair cache sharing and partitioning on a chip multiprocessor architecture. In Proceedings of the International Conference on Parallel Architectures and Compilation Techniques. 111-122.

LeE, J. W. ANd AsANovic, K. 2006. METERG: Measurement-based end-to-end performance estimation technique in QoS-capable multiprocessors. In Proceedings of the 12th IEEE Real-Time and Embedded Technology and Applications Symposium. 135-147.

Lin, J., Lu, Q., Ding, X., Zhang, Z., Zhang, X., ANd P.SAdayappan. 2008. Gaining insights into multicore cache partitioning: Briding the gap between simulation and real systems. In Proceedings of the 14th International Symposium on High Performance Computer Architecture. 367-378.

Luo, Y., Lubeck, O. M., Wasserman, H., Bassetti, F., and Cameron, K. W. 1998. Development and validation of a hierarchical memory model incorporating CPU- and memory-operation overlap model. In Proceedings of the 1st International Workshop on Software and Performance. $152-163$.

Magnusson, P. S., Christensson, M., Eskilson, J., Forsgren, D., Hallberg, G., Hogberg, J., Larsson, F., Moestedt, A., and Werner, B. 2002. Simics: A full system simulation platform. IEEE Computer 35, 2, 50-58.

Nesbit, K. J., Laudon, J., ANd Smith, J. E. 2007. Virtual private caches. In Proceedings of the 34th International Symposium on Computer Architecture. 57-68.

Papazoglou, M. P. and Georgakopoulos, D. 2003. Service-oriented computing: Introduction. Comm. ACM 46, 10, 24-28.

Qureshi, M. K., Lynch, D. N., Mutlu, O., ANd Patt, Y. N. 2006. A case for MLP-aware cache replacement. In Proceedings of the 33rd Annual International Symposium on Computer Architecture. 167-178.

QuReshi, M. K. AND PAtT, Y. N. 2006. Utility-based cache partitioning: A low-overhead, highperformance, runtime mechanism to partition shared caches. In Proceedings of the 39th Annual IEEE / ACM International Symposium on Microarchitecture. 423-432.

ACM Transactions on Architecture and Code Optimization, Vol. 7, No. 3, Article 14, Pub. date: December 2010. 
Rafique, N., Lim, W., AND Thottethodi, M. 2006. Architectural support for operating systemdriven CMP cache management. In Proceedings of the 15th International Conference on Parallel Architectures and Compilation Techniques. 2-12.

Rajkumar, R., Juvva, K., Molano, A., ANd Oikawa, S. 1998. Resource kernel: A resource-centric approach to real-time and multimedia systems. In Proceedings of the SPIE / ACM Conference on Multimedia Computing and Networking. 150-164.

RAJKumar, R., LeE, C., LEHOCZKY, J., AND SiewioReK, D. 1997. A resource allocation model for QoS management. In Proceedings of the 18th IEEE Real-Time Systems Symposium. 298-307.

Ranganathan, P. AND Jouppi, N. 2005. Enterprise IT trends and implications for architecture research. In Proceedings of the 11th International Symposium on High Performance Computer Architecture. 253-256.

Standard Performance Evaluation Corporation. 2006. Spec cpu2006. http://www.spec.org/ cpu2006.

Subramanian, R., Smaragdakis, Y., and LoH, G. H. 2006. Adaptive caches: Effective shaping of cache behavior to workloads. In Proceedings of the 39th Annual IEEE / ACM International Symposium on Microarchitecture. 385-396.

SuH, G. E., Devadas, S., AND Rudolph, L. 2001. Analytical cache models with applications to cache partitioning. In Proceedings of the 15th International Conference on Supercomputing. ACM Press, $1-12$.

Wang, J., Zhou, S., ANd Ahmed, W. 1993. Lsbatch: A distributed load sharing batch system. Tech. rep. CSRI-286, Univ. of Toronto.

YeH, T. Y. AND Reinman, G. 2005. Fast and fair: Data-stream quality of service. In Proceedings of the International Conference on Compilers, Architectures and Synthesis for Embedded Systems. 237-248.

Received March 2009; revised September 2009; accepted April 2010 